NASA Technical Meinorandum 88926

$\rightarrow+$ AIAA-87-0355

\title{
A Generalized Procedure for Constructing an Upwind-Based TVD Scheme
}

\author{
(AASA-TE-88926) A GEEEAIIZEI FECCEDORE FOR \\ N87-241ミ2 \\ CCASTBUCIING AN UEHINL EASED TVL SCHBHE \\ (NASA) 27 P Avail: MIS EC A03/BF A01 \\ CSCL $12 \mathrm{~A}$ \\ $\begin{array}{ll}\text { Unclas } \\ \text { 11/64 } & 0076757\end{array}$
}

Meng-Sing Liou

Lewis Research Center

Cleveland, Ohio

Prepared for the

25th Aerospace Sciences Meeting

sponsored by the American Institute of Aeronautics and Astronautics

Reno, Nevada, January 12-15, 1987

\section{nusn}


Meng-Sing Liou

National Aeronautics and Space Administration

Lewis Research Center

Cleveland, Ohio 44135

\section{Abstract}

A generalized formulation for constructing second- and higher-order accurate TVD (total vari. ation diminishing) schemes is presented. A given scheme is made TVD by limiting antidiffusive flux differences with some nonlinear functions, so-called limiters. The general idea of the formulation and its mathematical proof of Harten's TVD conditions is shown by applying the Lax-Wendroff method to a scalar nonlinear equation and constant coefficlent system of conservation laws. For the system of equations, we derive several definitions for the argument used in the limiter function and present their performance in numerical experiments. Then the formulation is formally extended to the nonlinear system of equations. It is demonstrated that use of the present procedure allows easy conversion of existing central or upwind, and second-or higher-order differencing schemes so as to preserve monotonicity and to yield physically admissable solutions. The formulation is simple mathematically as well as numerically; neither matrix-vector multiplication nor Riemann solver is required. Roughly twice as much computational effort is needed as compared to conventional scheme. Although the notion of TVD is based on the initial value problem, application to the steady Euler equations of the formulation is also made. Numerical examples including various ranges of problems show both time- and spatial-accuracy in comparison with exact solutions.

\section{Introduction}

Recently several second-order, nonoscillatory schemes have been proposed for solving hyperbolic system of conservation laws, as a means to obtain accurate weak solutions. (hereafter the term "second-order accuracy" is applied only in the region excluding point of extrema in fluxes) See, for examp le, van Leer ${ }^{1}$, Harten ${ }^{2}, \mathrm{Roe}^{3}$, and Osher and Chakravarthy ${ }^{4}$. Sweby ${ }^{5}$ presented a unified formulation in which these independently proposed schemes bolled down to using different forms of flux limiters due originally to van Leer ${ }^{1}$.

Harten ${ }^{2}$ introduced the notion of TVD (total variation diminishing) and gave sufficient conditions for a class of 5-point, explicit schemes to be second-order accurate as well as TVD. It is easy to show that all second-and higher-order schemes can always be recast as a combination of some first-order, stable scheme with higher-order terms. The first-order scheme is too dissipative, especially crude in the regions of high gradients. Consequently these higher-order terms are added to take away excessive diffusivity, hence appropri. ately called antidiffusive terms by Boris and Book6, while maintaining stability. It is wellknown that conventional second- (or higher) order, shock-capturing schemes produce spurious oscillations near discontinuties and generally require considerable numerical dissipation. Thus, the basic schemes must be altered (e.g., by limiting flux differences in our case) to meet such requirements as (1) yielding monotonic and sharp representation of jumps, and (2) satisfying the entropy condition so as to automatically select physically relevant solutions. Van Leer first successfully attacked this problem and arrived at his second. order scheme. The crux of the matter is to form a nonlinear combination of the underlying first-order scheme and the remaining antidiffusive terms. Hence we view that in second-and higher-order TVD schemes the antidiffusive terms basically play seemingly uncompromising rales of both increasing accuracy and diminishing the total variation. We also remark that all TVD schemes cited above use some form of an upwinding scheme as the underlying first-order scheme. In this paper we apply this principle to formulate our TVD scheme with emphasis on nonlinear systems of conservation laws, namely Euler equations. This approach appears natural and straightforward for extension to multispace dimensions. Although rigorous mathematical procedures are followed to ensure Harten's TVD conditions, the actual implementation for solving the multidimensional Euler equations is quite simple and general. In numerical tests we even carried over the same difference schemes, proved to be TVD in the hyperbolic system, directly to the steady Euler equations whose type is not known. The same characteristics as that obtained by solving unsteady equations were found.

Section 2 shows the present formulation for the nonlinear scalar equation. The procedure follows closely to that of Sweby 5 , especially in the TVD proof, but they differ in the definition of a basic first-order scheme. The entropy condition is also examined. Section 3 shows the extension to the one-dimensional system of conservation laws and the corresponding TVD property for a linearized system. Finally, a description of the generization to the Euler equations of gas dynamics is given in Section 4. Application to steady equations, use of both explicit and implicit schemes, central and upwinding differences are also included.

\section{Second-order TVD Scheme for Nonlinear Scalar Equation}

To faclliate understanding of the present formulation, first we consider the numerical solution of the scalar nonlinear conservation law in one space

$$
\begin{gathered}
u_{t}+f_{x}(u)=0, \quad t>0, \quad-\infty<x<\infty \\
u(x, 0)=u_{0}(x)
\end{gathered}
$$

For Eq. (2.1) to be of hyperbolic type, the flux function $f(u)$ has a real derivative $a=d f / d u$ with characteristic curve described by $d x / d t=a$.

Suppose a second- or higher-order (centered or one-sided biased) differencing scheme is given (e.g., Eq. (2.5b)), the present formulation makes 
it TVD-satisfying by the following steps:

Form a first-order, upwind differencing,

(Eq. (2.5a)); (2) Carry out subtraction of the differencings and obtain the remaining terms, called antidiffusive terms, (Eq. (2.6)); (3) Limit the antidiffusive terms, (Eq. (2.7)), using the so-called flux-1imiter function ${ }^{3}$; ${ }^{*}$ and (4) Determine the bounds of the limiter from Harten's con. ditions $^{2}$, as well as specify its function forms.

The upwinding is conveniently achieved by splitting a(u) (a is real function) into "+" and "-" parts,

$$
a=a^{+}+a^{-}
$$

where

$$
a^{+} \geq 0, \quad a^{-} \leq 0
$$

Thus the flux $f$ is split accordingly by requiring:

$$
\begin{aligned}
& \text { (i) } f=f^{+}+f^{-} \\
& \text {(ii) } a^{+}=\left(f^{+}\right)_{u}, \quad a^{-}=\left(f^{-}\right)_{u}
\end{aligned}
$$

We note that how the splitting (not unique) is done is irrelevant in the present analysis. It is needed only if an actual calculation is made. We also remark that Roe's flux difference splitt ing ${ }^{3}$ can be applied equally well, as will become clear later.

Let us use the notation

$$
\begin{aligned}
& \Delta^{+}()_{j}=()_{j+1}()_{j} \\
& \Delta^{-}()_{j}=()_{j}-()_{j-1}
\end{aligned}
$$

We now approximate $f_{x}$ :

$$
\begin{aligned}
f_{x} & =\left(\Delta^{+} f^{-}+\Delta^{-} f^{+}\right) / \Delta x \text {, first-order upwinding } \\
& =\left(\Delta^{+} f+\Delta^{-} f\right) / 2 \Delta x \text {, centra } 7 \text {-differencing }
\end{aligned}
$$

Subtracting Eqs. (2.5a) from (2.5b), we have the antidiffusive term

$$
\Delta^{-}\left(\Delta^{+} f^{+}-\Delta^{+} f^{-}\right) / 2 \Delta x
$$

The flux differences $\Delta^{+} f \pm$ are limited by assigning the limiter functions $\varphi^{+}$and $\varphi^{-}$respectively, and we write

$$
f_{x}=\left(\Delta^{+} f^{-}+\Delta^{-} f^{+}\right) / \Delta x+\Delta^{-}\left(\varphi^{+} \Delta^{+} f^{+}-\varphi^{-} \Delta^{+} f^{-}\right) / 2 \Delta x
$$

*Since it is the flux differences, rather than fluxes themselves, that are limited, it might be more appropriate to call this the fluxdifference limiter.
It is noted that allowing different functions $\varphi^{+}$ and $\varphi^{-}$to be associated with $f^{+}$and $f^{-}$is essential for the proof of TVO.

Now, the integration scheme for Eq. (2.1) can be applied. To illustrate some preliminaries of TVD and Harten's conditions, we consider the first-order Euler explicit scheme with the use of upwind differencing, Eq. (2.5a). Then we have

$$
u_{j}^{n+1}=u_{j}^{n}-\lambda\left(\Delta^{+} f_{j}^{-}+\Delta^{-} f_{j}^{+}\right)^{n}, \quad \lambda=\Delta t / \Delta x
$$

or

$$
u_{j}^{n+1}=u_{j}^{n}-\lambda\left(\frac{\Delta^{+} f_{j}^{-}}{\Delta^{+} u_{j}} \Delta^{+} u_{j}+\frac{\Delta^{-} f_{j}^{+}}{\Delta^{-} u_{j}} \Delta^{-} u_{j}\right) n
$$

This is rewritten in a general form

$$
u_{j}^{n+1}=u_{j}^{n}-c_{j-1}^{+} \Delta^{-} u_{j}^{n}+c_{j}^{-} \Delta^{+} u_{j}^{n}
$$

We now define the total variation of the mesh solution $u$ to be

$$
\operatorname{TV}(u)=\sum_{j}\left|\Delta^{+} u_{j}\right|
$$

A numerical scheme is said to be TVD if

$$
\operatorname{TV}\left(u^{n+1}\right) \leq \operatorname{TV}\left(u^{n}\right)
$$

Harten $^{2}$ gave sufficient conditions for Eq. (2.9) to be TVO.

Lemma (Harten). Let the coefficients $c^{+}$and $\mathrm{C}^{-}$in Eq. (2.9) satisfy the inequalities

$$
\begin{gathered}
c_{j}^{+} \geq 0 \\
c_{j}^{-} \geq 0 \\
c_{j}^{+}+c_{j}^{-} \leq 1
\end{gathered}
$$

then the scheme Eq. (2.9) is TVD.

For smooth functions $f \pm$, we have

$$
\begin{gathered}
\frac{\Delta^{+} f_{j}^{+}}{\Delta^{+} u_{j}}=a_{j}^{+}+0\left(\Delta^{+} u_{j}\right) \\
\frac{\Delta^{+} f_{j}^{-}}{\Delta^{+} u_{j}}=a_{j}^{-}+0\left(\Delta^{+} u_{j}\right) \\
\Delta^{+} u_{j}=0(\Delta x)
\end{gathered}
$$

where

Hence Eqs. (2.12a) and (2.12b) are satisfied by Eq. (2.2b) and the third inequality, Eq. (2.12C) requitres 


$$
\lambda\left(\frac{\Delta^{+} f_{j}^{+}}{\Delta^{+} u_{j}}-\frac{\Delta^{+} f_{j}^{-}}{\Delta^{+} u_{j}}\right) \leq 1
$$

or

$$
\lambda\left|a_{j}\right|=\lambda\left(a_{j}^{+}-a_{j}^{-}\right) \leq 1
$$

which is identical to the CFL stability condition.

Roe $^{3}$ proposed another type of splitting in which the flux differences are expressed in terms of the mean value of the Jacobian matrix. The Jacobian matrix ( $|x|$ in scalar case) is required to satisfy

$$
\begin{aligned}
& \text { (i) } f(u)-f(v)=A(u, v)(u-v) \\
& \text { (ii) } A(u, u)=f_{u}(u)=A(u)
\end{aligned}
$$

(11i) $A(u, v)$ has real eigenvalues and a complete set of eigenvectors

$(2.14 c)$

Roe $^{3}$ has constructed a linearization of the form Eq. (2.14a) having these properties. Harten and Lax 7 show that such a Roe-type 11 nearlzation exists as long as there is an entropy function. Hence, from Eqs. (2.14) we can write

$$
\begin{array}{r}
\Delta^{-} f_{j}^{+}=f^{+}\left(u_{j}\right)-f^{+}\left(u_{j-1}\right)=a^{+}\left(u_{j}, u_{j-1}\right)\left(u_{j}-u_{j-1}\right) \\
=a^{+}\left(u_{j}, u_{j-1}\right) \Delta^{-} u_{j} \quad(2.15 a) \\
\begin{aligned}
\Delta^{+} f_{j}^{-}=f^{-}\left(u_{j+1}\right)-f^{-}\left(u_{j}\right) & =a^{-}\left(u_{j}, u_{j+1}\right)\left(u_{j+1}-u_{j}\right) \\
& =a^{-}\left(u_{j}, u_{j+1}\right) \Delta^{+} u_{j} \quad(2.15 b)
\end{aligned}
\end{array}
$$

where $a^{+}\left(u_{j}, u_{j-1}\right) \geq 0, a^{-}\left(u_{j}, u_{j+1}\right) \leq 0$. For scalar equation Eq. (2.1), the property, Eq. (2.14c) is clearly satisfied. Thus the TVo conditions Eq. (2.12) can be ensured by this construction of $\mathrm{a}^{+}$and $\mathrm{a}^{-}$. This concludes the TVD consideration of the first-order scheme Eq. (2.8).

Since first-order schemes contain too much dissipation, often obscuring the physics, we wish to convert them to TVD-satisfying, second-order accurate (both in space and time) schemes by adding limited antidiffusive terms. Let us consider the one-step, Lax-Wendroff scheme

$$
u_{j}^{n+1}=u_{j}^{n}-\Delta t f_{x}^{n}+0.5 \Delta t^{2}\left(a f_{x}\right)_{x}^{n}+0\left(\Delta t^{3}\right)
$$

The third term on the RHS of Eq. (2.16) is of second-order accuracy and is considered as a part of the antidiffusive terms. We now can apply limiters to yield:

$$
\begin{aligned}
\left(a_{x}\right)_{x}=\Delta^{-}\left\{\varphi_{j}^{+} a_{j+1 / 2} \Delta^{+} f_{j}^{+}\right. \\
\\
\left.+\varphi_{j+1}^{-} a_{j+1 / 2} \Delta^{+} f_{j}^{-}\right\} / \Delta x^{2}
\end{aligned}
$$

$$
a_{j+1 / 2}=\left(a_{j}+a_{j+1}\right) / 2
$$

Hence we find

$$
\begin{aligned}
u_{j}^{n+1}=u_{j}^{n} & -\lambda\left(\Delta^{-} f_{j}^{+}+\Delta^{+} f_{j}^{-}\right)^{n} \\
& -0.5 \lambda \Delta^{-}\left\{\varphi_{j}^{+}\left(1-\lambda a_{j+1 / 2}\right) \Delta^{+} f_{j}^{+}\right. \\
& \left.-\varphi_{j+1}^{-}\left(1+\lambda a_{j+1 / 2}\right) \Delta^{+} f_{j}^{-}\right\}
\end{aligned}
$$

After rearranging, Eq. (2.18) becomes

$$
\begin{aligned}
& u_{j}^{n+1}=u_{j}^{n}-\lambda\left\{1+0.5\left[\left(1-\lambda a_{j+1 / 2}\right) \varphi_{j}^{+} \frac{\Delta^{+} f_{j}^{+}}{\Delta^{-} f_{j}^{+}}\right.\right. \\
& \left.\left.-\left(1-\lambda a_{j-1 / 2}\right) \varphi_{j-1}^{+}\right]\right\}^{n}\left(\frac{\Delta^{-} f_{j}^{+}}{\Delta^{-} u_{j}}\right) \Delta^{-} u_{j}^{n} \\
& +\lambda\left\{1-0.5\left[\left(1+\lambda a_{j+1 / 2}\right) \varphi_{j+1}^{-}\right.\right. \\
& \left.\left.-\left(1+\lambda a_{j-1 / 2}\right) \varphi_{j}^{-} \frac{\Delta_{j}^{-} f_{j}^{-}}{\Delta^{+} f_{j}^{-}}\right]^{n}\right)\left(-\frac{\Delta^{+} f_{j}^{-}}{\Delta^{+} u_{j}}\right)^{-} \Delta^{+} u_{j}^{n}
\end{aligned}
$$

The underlying idea of the above arrangement is to separately group "+" and "-" fluxes. This turns out to be an important step for the TVD proof. Now let

$$
\begin{aligned}
& c_{j-1}^{+}=\lambda\left(\frac{\Delta^{-} f_{j}^{+}}{\Delta^{-} u_{j}}\right)\left\{1+0.5\left(1-\lambda a_{j-1 / 2}\right)\right. \\
& \left.\left[\frac{1-\lambda a_{j+1 / 2}}{1-\lambda a_{j-1 / 2}} \frac{\Delta^{+} f_{j}^{+}}{\Delta_{j}^{+}} \varphi_{j}^{+}-\varphi_{j-1}^{+}\right]\right\} \\
& c_{j}^{-}=\lambda\left(-\frac{\Delta^{+} f_{j}^{-}}{\Delta^{+} u_{j}}\right)\left\{1-0.5\left(1+\lambda a_{j+1 / 2}\right)\right. \\
& \left.\left[\varphi_{j+1}^{-}-\frac{1+\lambda a_{j-1 / 2}}{1+\lambda a_{j+1 / 2}} \frac{\Delta^{-} f_{j}^{-}}{\Delta^{+} f_{j}^{-}} \varphi_{j}^{-}\right]\right\}
\end{aligned}
$$

This provides us with an obvious choice for the functional relation for the limiter function, i.e.,

$$
\varphi_{j}^{ \pm}=\varphi\left(r_{j}^{ \pm}\right)
$$

where

$$
r_{j}^{+}=\frac{\Delta^{-} f_{j}^{+}\left(1-\lambda a_{j-1 / 2}\right)}{\Delta^{+} f_{j}^{+}\left(1-\lambda a_{j+1 / 2}\right)}, r_{j}^{-}=\frac{\Delta^{+} f_{j}^{-}\left(1+\lambda a_{j+1 / 2}\right)}{\Delta^{-} f_{j}^{-}\left(1+\lambda a_{j-1 / 2}\right)}
$$

where 


$$
\begin{aligned}
& c_{j-1}^{+}=\lambda\left(\frac{\Delta^{-} f_{j}^{+}}{\Delta^{-} u_{j}}\right)\left\{1+0.5\left(1-\lambda a_{j-1 / 2}\right)\left(\frac{\varphi_{j}^{+}}{r_{j}^{+}}-\varphi_{j-1}^{+}\right)\right\} \\
& c_{j}^{-}=\lambda\left(-\frac{\Delta^{+} f_{j}^{-}}{\Delta^{+} u_{j}}\right)\left\{1-0.5\left(1+\lambda a_{j+1 / 2}\right)\left(\varphi_{j+1}^{-}-\frac{\varphi_{j}^{-}}{r_{j}^{-}}\right)\right\}
\end{aligned}
$$

We note that Eqs. (2.19) to (2.21) are identical in form to that obtained by Sweby 5 . Differences only appear in the detail; here we use the whole $a$, rather than $a^{+}$and $a^{-}$in $\left(r^{+}, c^{+}\right)$and $\left(r^{-}, C^{-}\right)$. Since $\Delta^{-} f^{+} / \Delta^{-} u \geq 0$ and $\left(-\Delta^{+} f^{-} / \Delta^{+} u\right) \geq 0$, Eq5. (2.12a) and (2.12b) are satisfied by requiring

$$
\left|\begin{array}{ll}
\varphi_{j}^{ \pm} & \\
r_{j}^{ \pm} & \varphi_{j \mp 1}^{ \pm}
\end{array}\right|=\Phi \leq 1
$$

and $\mathrm{Eq} .(2.12 \mathrm{C})$ leads to the condition

$$
\frac{\Delta^{+}}{\Delta^{+} u}=f^{+} L \leq 1
$$

or

$$
\lambda|\mathrm{a}| \leq 1
$$

Note that in Eq. $(2.23 \mathrm{c})$, the TVD condition Eq. $(2.12 \mathrm{C})$ puts the same limit on the CFL number as the stability condition does. We note that Sweby 5 found: (1) the CFL number must be reduced to achieve the TVD conditions and (2) $\Phi \leq 2$. The differences between the present results and Sweby's are obviousiy due to the differences in formulation.

We turn now to the determination of the bounds for the limiter functions. Allowing maximum diffusivity to occur only in the first-order upwinding scheme requires

$$
\varphi^{ \pm} \geq 0 \quad \text { for any } r^{ \pm}
$$

For all TVD schemes using limiter-type formulations, an additional constraint is imposed,

$$
\varphi^{t}=0, \quad r^{ \pm} \leq 0
$$

That is, a first-order scheme reverts as flux differences at neighboring points are of opposite signs, i.e., at point of extrema in flux. Hence second-order accuracy is lost locally. This appears a price that has to be paid to make the limiter-type scheme TVD. Recent development of the so-called ENO(Essentially NonOscillatory) scheme ${ }^{8}$ remedies this shortcoming, but allows some "controlled" oscillations.

$$
0 \leq\left(\frac{\varphi}{r}, \varphi\right) \leq 1
$$

Next, we illustrate the entropy condition with a specific example. Let $f(u)=u^{2} / 2$, hence $a=u$. An obvious splitting of a gives

$$
a^{ \pm}=(u \pm|u|) / 2
$$

and

$$
f^{ \pm}=a^{ \pm} u / 2
$$

Note that since $f$ is a homogeneous function of degree 2, it is easy to see that $f \pm$ have continuous first derivatives. Let the initial condition

$$
u_{0}= \begin{cases}u_{L}, & x<0 \\ u_{R,} & x>0\end{cases}
$$

and

$$
f\left(u_{L}\right)=f\left(u_{R}\right)
$$

The exact solutions are shown in Figs. 1.

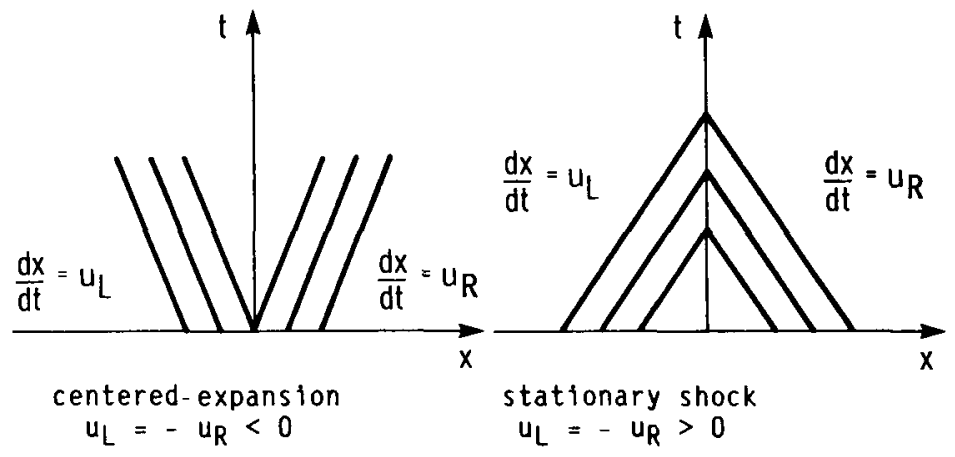

Figure 1 Exact (similarity) solution of Burgers' equation with initial conditions, Eqs. (2.29).

In the case, $u_{L}<0$, since the expansion jump is not physically admissable, hence centered-expansion fans give rise to unsteady solutions. On the other hand, the case $u_{L}>0$ allows a stationary shock solution for $t>0$. It is noted that Roe's original flux-difference-split scheme and Murman-Cole's scheme for transonic potential equation admit expansion shocks. (See e.g., Harten et al. ${ }^{9}$ )

In what follows, we see the performance of the first-order scheme Eq. (2.8) along with the splitting Eqs. (2.27) and (2.28). For calculation purpose, we assign

$$
u_{j}(t=0)=\left\{\begin{array}{l}
u_{L}, j \leq-1 \\
u_{R}, j \geq 0
\end{array}\right.
$$

and take time step, $\lambda\left|u_{L}\right|=1.0$.

(i) Expansion, $u_{L}=-u_{R}<0$ 
(1) Expansion, $u_{L}=-u_{R}<0$

$\begin{array}{ccccccc}\text { time step } & u_{-} & u_{-2} & u_{-1} & u_{0} & u_{1} & u_{2} \\ \Delta t & u_{L} & u_{L} & u_{L} / 2 & u_{R} / 2 & u_{R} & u_{R} \\ 2 \Delta t & u_{L} & 5 u_{L} / 8 & 3 u_{L} / 8 & 3 u_{R} / 8 & 5 u_{R} / 8 & u_{R} \\ 3 \Delta t & 89 u_{L} / 128 & u_{L} / 2 & 39 u_{L} / 128 & 39 u_{R} / 128 & u_{R} / 2 & 89 u_{R} / 128 \\ \vdots & & & & & & \end{array}$

Clearly the scheme does not admit an expansion shock. The expansions are centered about the midpoint between initial jumps, $x=-0.5 \Delta x$, and spread over $2 n$ grid points at $t=n \Delta t$.

$$
\begin{array}{ccccc}
\text { Shock, } u_{L}=-u_{R}>0 \\
\text { time step } & u_{-2} & u_{-1} & u_{0} & u_{1} \\
\Delta t & u_{L} & 0.5 u_{L} & 0.5 u_{R} & u_{R} \\
2 \Delta t & u_{L} & 0.75 u_{L} & 0.75 u_{R} & u_{R} \\
3 \Delta t & u_{L} & 0.688 u_{L} & 0.688 u_{R} & u_{R} \\
4 \Delta t & u_{L} & 0.715 u_{L} & 0.715 u_{R} & u_{R} \\
5 \Delta t & u_{L} & 0.703 u_{L} & 0.703 u_{R} & u_{R} \\
6 \Delta t & u_{L} & 0.708 u_{L} & 0.708 u_{R} & u_{R} \\
: & u_{L} & 0.707 u_{L} & 0.707 u_{R} & u_{R}
\end{array}
$$

We see that the shock wave is stationary and is represented with two interior points. In general, both Gudonov's and Roe's schemes allow a stationary shock with one interior state while the Engquist-0sher scheme admits two states. (See also van Leer ${ }^{10}$ ) Since Roe's scheme also admits expansion shocks, the Gudonov scheme stands as the best for the representation of a stationary shock connecting the states Eq. (2.30), at least for $f(u)=u^{2} / 2$.

For system of equations, the split-flux method is shown to satisfy the entropy inequality 9 , and is thereby capable of selecting physically admissable solutions. A contact discontinuity, however, will be smeared over a large extent, as seen later in section 4 . Harten ${ }^{2}$ suggests use of artificial compression to further sharpen the contact discontinuity. However some care must be taken in a region of expansion so that entropy condition is not violated, see Yee et al.11

We now express the present TVD scheme in terms of numerical fluxes $\widetilde{f}_{j \pm 1 / 2}$ in the equation

$$
u_{j}^{n+1}=u_{j}^{n}-\lambda\left(\tilde{f}_{j+1 / 2}-\tilde{f}_{j-1 / 2}\right)
$$

Rewriting Eq. (2.8a), the first-order upwind scheme yields

$$
\tilde{f}_{j+1 / 2}=\left(f_{j+1}+f_{j}-\Delta^{+} f_{j}^{*}\right) / 2, f^{\star}=f^{+}-\underset{f}{-}
$$

We note that Eq. (2.32) is not the CourantIsacsson-Rees scheme

$$
\tilde{f}_{j+1 / 2}=\left(f_{j+1}+f_{j}-\left|a_{j+1 / 2}\right| \Delta+u_{j}\right) / 2
$$

where $\left|a_{j+1 / 2}\right|$ is a mean value given by $\Delta^{+} f_{j} / \Delta^{+} u_{j}$. Hence the case described by Eq. (2.29) will give rise to $a_{j \pm 1 / 2}=0$ and result in a stationary expansion shock, violating the entropy condition.

After some algebraic manipulation, the second-order scheme Eq. (2.19) gives

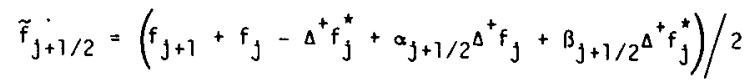

where

$$
\begin{aligned}
& \alpha_{j+1 / 2}=\left\{\varphi_{j}^{+}\left(1-\lambda a_{j+1 / 2}\right)-\dot{\varphi}_{j+1}^{-}\left(1+\lambda a_{j+1 / 2}\right)\right\} / 2 \\
& \beta_{j+1 / 2}=\left\{\varphi_{j}^{+}\left(1-\lambda a_{j+1 / 2}\right)+\varphi_{j+1}^{-}\left(1+\lambda a_{j+1 / 2}\right)\right\} / 2
\end{aligned}
$$

The last two terms in Eq. (2.34) obviously contribute to second-order accuracy and are the antidiffusion terms that also maintain TVD conditions.

Remark 1. - Note that $1+\lambda a j+1 / 2 \geq 0$ for stability, hence $B_{j+1 / 2}$ satisfies the inequality

$$
1 \geq \beta_{j+1 / 2} \geq 0, \quad \text { if } \quad 0 \leq \varphi_{j}^{ \pm} \leq 1
$$

and is of opposite sign to the third term on RHS of Eq. (2.34). Thus, it is clear that the present and other TVD schemes share the common feature in which the higher-order TVO procedure amounts to adding more sophisticated antidiffusion terms to a basic lower-order scheme which itself is a TVD scheme.

Remark 2. - On the other hand, it is more advantageous to think of it as adding diffusive terms to an underlying higher-order, non-TVD scheme so that TVD conditions are enforced. The one-step Lax-Wendroff scheme defines

$$
\tilde{f}_{j+1 / 2}^{L W}=\left(f_{j+1}+f_{j}-\lambda a_{j+1 / 2} \Delta f_{j}\right) / 2
$$

in Eq. (2.37). Rewriting Eq. (2.34) gives

$\left.\tilde{f}_{j+1 / 2}=\tilde{f}_{j+1 / 2}^{L W}-\left\{\left(1-B_{j+1 / 2}\right) \Delta^{+} f_{j}^{*}-\left(a_{j+1 / 2}+\lambda a_{j+1 / 2}\right) \Delta \Delta_{j}\right\}\right) / 2$

where it can be shown

for $0 \leq \varphi^{ \pm} \leq 1$. The last bracket in Eq. (2.38) as substituted in $\widetilde{f}_{\mathfrak{j}+1 / 2}-\widetilde{f}_{\boldsymbol{j}-1 / 2}$ is seen to yield a nonlinear second-order central difference. 
Remark 3. - Note that $\alpha_{j+1 / 2} \Delta^{+} f_{j}$ and $\left(1-B_{j+1 / 2}\right) \Delta^{+} f_{j}^{*}$ in Eq. (2.34) correspond to $\left(g_{j}+g_{j+1}\right)$ and $Q_{j+1 / 2} \Delta^{+} u_{j}$ in Harten's secondorder scheme. 2,11

Remark 4. - For smooth solutions of Eq. (2.1), we have $r^{ \pm}=1+0\left(\left|\Delta^{ \pm} u\right|\right)$ and $\varphi^{ \pm}=1+0\left(\left|\Delta^{ \pm} u\right|\right)$. It is easy to show that

$$
\widetilde{f}_{j+1 / 2}=\widetilde{f}_{j+1 / 2}^{L W}+0\left(\left|\Delta^{ \pm} u\right|^{2}\right)
$$

hence Eqs. (2.19) or (2.38) is second-order accurate.

\section{System of Conservation Laws- one-oimensional Equations}

In this section we describe how to extend the procedure developed for the scalar equation, namely upwinding combined with the notion of $11 \mathrm{~m}$ iters, to systems of conservation laws. To show the extension, we will exploit the fact that in one space dimension, the system is hyperbolic and hence is diagonalizable. As the system is decoupled, the mathematical procedure essentially follows what has been described in Section 2 . But it is less clear as to how to define the argument $r$ for the limiter $\varphi$, which is a key element in the limiter-type schemes.

Let us consider the one-dimensional system

$$
\begin{gathered}
U_{t}+F_{x}(U)=0, \quad t>0 \\
U(x, 0)=U_{0}(x)
\end{gathered}
$$

Here $U$ and $F(U)$ are column vectors of $m$ components, the flux vector has a Jacobian matrix

$$
A(U)=\frac{\partial F(U)}{\partial U}
$$

Eq. (3.1) is rewritten as

$$
U_{t}+A(U) U_{x}=0
$$

To complete the extension and the proof of TVD for the system, we need to make the following as sumptions: ${ }^{2}$

(1) A has real eigenvalues and a complete set of eigenvectors, hence $A$ is diagonalizable,

(2) A is constant.

Let $S$ be a matrix, the columns of which are the right eign evectors of $A$. Then

$$
S^{-1} A S=\Lambda
$$

where $\Lambda$ is a diagonal matrix

$$
\Lambda_{i j}=a_{i j} \delta_{i j}, \quad 1 \leq i, j \leq m
$$

Since $\Lambda$ is real, we can write

$$
\begin{array}{r}
\Lambda=\Lambda^{+}+\Lambda^{-}, \quad a_{i}=a_{i}^{+}+a_{i}^{-} \\
\Lambda^{ \pm}=(\Lambda \pm|\Lambda|) / 2, \quad\left|\Lambda_{i j}\right|=\left|a_{i}\right| \delta_{i j}
\end{array}
$$

Consequently A is split as

$$
\begin{aligned}
& A=A^{+}+A^{-} \\
& A^{ \pm}=S \Lambda^{ \pm} S^{-1}
\end{aligned}
$$

We note that to arrive at the split in matrix $A$, Eq. (3.7) the only condition used is that $A$ is diagonalizable. We have as yet made no connection with the flux vector $F$.

Now let $A$ be constant, hence $S$ and $\Lambda$ are constant. From Eq. (3.2) we have

$$
F=A U
$$

for any $U$. With the aid of Eq. (3.7), Eq. (3.8) gives a split in $F$

$$
\begin{aligned}
& F=F^{+}+F^{-} \\
& F^{ \pm}=A^{ \pm} U
\end{aligned}
$$

Here we remark that as a result of assumption (2), $F$ and $F^{ \pm}$are homogeneous functions of degree one. On the other hand, for the nonlinear system, specifically the Euler equations, Steger and Warming 12 need to rely upon the homogeneous property of $F$ to accomplish the flux splitting.

Employing the assumption (1), we define a vector $W$ by

$$
S^{-1} U=W, \quad W=\left\{w^{\ell}: \ell=1, m\right\}
$$

Hence

$$
\begin{aligned}
S^{-1} F & =\Lambda W \\
S^{-1} F^{ \pm} & =\Lambda^{ \pm} W
\end{aligned}
$$

Following the same procedure described in Section 2, the spatial derivatives which appeared in the Lax-Wendroff scheme are now approximated by

$$
F_{x}=\frac{\left(\Delta^{+} F_{j}^{-}+\Delta^{-} F_{j}^{+}\right)}{\Delta x}+\Delta^{-}-\left(\varphi_{j}^{+} \Delta^{+} F_{j}^{+}-\frac{\left.\varphi_{j+7^{-}}^{-} F_{j}^{+}\right)}{2 \Delta x}\right.
$$

and

$$
\left.\left(A F_{x}\right)_{x}=\Delta^{-} \frac{A_{j+1} / 2\left(\varphi_{j}^{+} \Delta^{+} F_{j}^{+}+\varphi_{j+1}^{-} \Delta^{+} F_{j}^{-}\right)}{\Delta x^{2}}\right)
$$

Here $\varphi^{ \pm}$are scalar functions, to be determined later. Then we have 


$$
\begin{aligned}
U_{j}^{n+1}=U_{j}^{n}- & \lambda\left(\Delta^{-} F_{j}^{+}+\Delta^{+} F_{j}^{-}\right)^{n} \\
& -0.5 \lambda \Delta^{-}\left\{\varphi_{j}^{+}\left(I-\lambda A_{j+1 / 2}\right) \Delta^{+} F_{j}^{+}\right. \\
& \left.-\varphi_{j+1}^{-}\left(I+\lambda A_{j+1 / 2}\right) \Delta^{+} F_{j}^{-}\right\}
\end{aligned}
$$

Letting $\varphi^{ \pm}=0$ reduces Eq. (3.14) to the Euler explicit, first-order, upwind scheme

$$
U_{j}^{n+1}=U_{j}^{n}-\lambda\left(\Delta^{-} F_{j}^{+}+\Delta^{+} F_{j}^{-}\right)
$$

In the following proof of TVo conditions for a system, Eq. (3.14) is considered and we will treat Eq. (3.15) as a subset of Eq. (3.14), although the second-order accurate TVO scheme is really built on the first-order scheme, a point brought out in the previous section. Premultiplying $\mathrm{Eq} .(3.14)$ with $\mathrm{S}^{-}$and using Eqs. (3.10) to $(3.11)$, one gets

$W_{j}^{n+1}=w_{j}^{n}-\lambda\left(\Lambda^{+} \Delta^{-} w_{j}+\Lambda^{-} \Delta^{+} W_{j}\right)^{n}$

$-0.5 \lambda \Delta^{-}\left\{\varphi_{j}^{+}(I-\lambda \Lambda) \Lambda^{+} \Delta^{+} W_{j}-\varphi_{j+1}^{-}(I-\lambda \Lambda) \Lambda^{-} \Delta^{+} W_{j}\right\} n$

Since $\Lambda^{ \pm}$are diagonal matrices and $\varphi_{j}$ 's are scalar functions, Eq. $(3.16)$ is a m-component system of decoupled equations,

$$
\begin{aligned}
& \left(w_{j}^{\ell}\right)^{n+1}=\left(w_{j}^{\ell}\right)^{n}-\lambda\left(a_{\ell}^{+} \Delta^{-} w_{j}^{l}+a_{\ell}^{-} \Delta^{+} w_{j}^{\ell}\right)^{n} \\
& -0.5 \lambda \Delta^{-}\left\{\varphi_{j}^{+}\left(1-\lambda a_{\ell}\right) a_{\ell}^{+} \Delta^{+} w_{j}\right. \\
& \left.-\varphi_{j+1}^{-}\left(1-\lambda a_{\ell}\right) a_{\ell}^{-} \Delta^{+} w_{j}\right\} n, \quad 1 \leq \ell \leq m
\end{aligned}
$$

This is identical to the constant-coefficlent version of the scheme of Eq. (2.19) for the nonlinear scalar equation if $\Delta^{F} f^{ \pm}=a^{ \pm} \Delta^{F} u$. Proof of TVD however departs here from that in Section 2. The most important thing to keep in mind is: the transformation (decoupling) is employed merely as a mechanism to ensure TVD, the ultimate goal is treating the system. Therefore, in the process of proving TVD conditions for Eq. $(3.17)$, definitions regarding $r$ and $\varphi(r)$ must not hinder the transformation back to the coupled system of Eq. $(3.14)$.

Dropping superscript " $\ell, " \mathrm{Eq} .(3.17)$ is rewritted as

$$
w_{j}^{n+1}=w_{j}^{n}-c_{j-1}^{+} \Delta^{-} w_{j}^{n}+c_{j}^{-} \Delta^{+} w_{j}^{n}
$$

with

$$
c_{j-1}^{+}=\lambda a^{+}\left\{1+0.5(1-\lambda a)\left[\frac{a^{+} \Delta^{+} w_{j}}{a^{+} \Delta^{-} w_{j}} \varphi_{j}^{+}-\varphi_{j-1}^{+}\right]\right\}
$$

$c_{j}^{-}=\left(-\lambda a^{-}\right)\left\{1-0.5(1+\lambda a)\left[\varphi_{j+1}^{-}-\frac{a^{-} \Delta^{-} w_{j}}{a^{-} \Delta^{+} w_{j}} \varphi_{j}^{-}\right]\right\}$

Here the trick is: associate all term having "+" eigenvalues with $\varphi^{+}$and those of " " eigenvalues with $\varphi^{-}$. Also, recognizing the fact that the transformation is possible only for those $w$ connected with $a_{\ell}$, it becomes clear that we must redefine $r$ for the system. An obvious choice is

$$
\begin{aligned}
& r_{j}^{+}=\frac{a^{+} \Delta^{-} w_{j}}{a^{+} \Delta^{+} w_{j}} \\
& r_{j}^{-}=\frac{a^{-} \Delta^{+} w_{j}}{a^{-} \Delta^{-} w_{j}}
\end{aligned}
$$

Eqs. (3.19) are rewritten as

$$
\begin{aligned}
& c_{j-1}^{+}=\left(\lambda a^{+}\right)\left\{1+0.5(1-\lambda a)\left(\frac{\varphi_{j}^{+}}{r_{j}^{+}}-\varphi_{j-1}^{+}\right)\right\} \\
& c_{j}^{-}=\left(-\lambda a^{-}\right)\left\{1-0.5(1+\lambda a)\left(\varphi_{j+1}^{-}-\frac{\varphi_{j}^{-}}{r_{j}^{-}}\right)\right\}
\end{aligned}
$$

Harten's TVO conditions Eqs. (2.12) are satisfied if

$$
\left|\frac{\varphi_{j}^{ \pm}}{r_{j}^{ \pm}}-\varphi_{j+1}^{ \pm}\right|=\Phi \leq 1
$$

and

$$
\lambda|a| \leq 1
$$

as Eq. (2.23). This completes the TVD proof for each component of the decoupled system.

If a solution procedure for Eq. (3.1) is constructed for the decoupled system Eq. (3.17), we see that the definitions of Eq. (3.20) require knowledge of the eigenvalues and eigenvectors of A (see Eqs. (3.4), (3.5) and (3.10)). This causes concerns not only in numerical operation counts but also in extention to multidimensions, because decoupling systems in multidimensions may be an extremely complex task if possible at all. Hence, the goal is to express $r^{ \pm}$in terms of simple variables e.g., $U$ or primitive variables, or preferably fluxes. The latter has the attractive property being able to naturally reduce to the scalar case.

We turn now to show a rational procedure to arrive at definitions of $r^{ \pm}$for systems of equations. Putting the superscript " $l$ "back in Eq. (3.20a), ( $r^{-}$is done similarly) but dropping subscript " $j$ ", we have 


$$
a_{\ell}^{+} \Delta^{-} w^{\ell}=r_{\ell}^{+} a_{\ell}^{+} \Delta^{+} w^{\ell}, \quad 1 \leq \ell \leq m
$$

or in matrix form

$$
\Lambda^{+} \Delta^{-} W=R^{+} \Lambda^{+} \Delta^{+} W
$$

where $R^{+}$is a diagonal matrix whose diagonal elements are $\{r\{: \ell=1, \mathrm{~m}\}$. Premultiplying both sides with's brings us back to the coupled system,

$$
A^{+} \Delta^{-} U=R^{+} A^{+} \Delta^{+} U
$$

With the aid of Eq. (3.9), we can relate fluxes to $r^{+}$

or

$$
\Delta^{-} F^{+}=R^{+} \Delta^{+} F^{+}
$$

$$
\Delta^{-} F_{\ell}^{+}=r_{\ell}^{+} \Delta_{\ell}^{+} F_{\ell}^{+}, \quad 1 \leq \ell \leq m
$$

Since the signs of the flux differences are key factor in determining the limiter function $\varphi$. use of norms must be ruled out. However, some kind of sum is needed in order to include influences from each component. Several possibilities immediately follow from Eqs. (3.26).

$$
\begin{aligned}
& \text { (a) } \sum_{\ell} r_{\ell}^{+} \Delta^{+} F_{\ell}^{+}=\sum_{\ell} \Delta^{-} F_{\ell}^{+} \\
& \text {(b) } \sum_{\ell} r_{\ell}^{+}\left(\Delta^{+} F_{\ell}^{+}\right)^{2}=\sum_{\ell}\left(\Delta^{-} F_{\ell}^{+}\right)\left(\Delta^{+} F_{\ell}^{+}\right) \\
& \text {(c) } \sum_{\ell} r_{\ell}^{+}\left|r_{\ell}^{+}\right|\left|\Delta^{+} F_{\ell}^{+}\right| \Delta^{+} F_{\ell}^{+}=\sum_{\ell}\left|\Delta^{-} F_{\ell}^{+}\right| \Delta^{-} F_{\ell}^{+}
\end{aligned}
$$

The last choice mimics the $L_{2}$-norm but retains the sign. We see that Eqs. (3.27) offer the possibility of using different weights for different components of $F$. Let all components be of equal woight, then from Eqs. (3.27) we can define:

$$
\text { (a) } r^{+}=\sum_{\ell} \Delta^{-} F_{\ell}^{+} / \sum_{\ell} \Delta^{+} F_{\ell}^{+}=\left\langle\Delta^{-} F^{+}, 1\right\rangle /\left\langle\Delta^{+} F^{+}, 1\right\rangle
$$

(b) $r^{*}=\sum_{l}\left(\Delta^{-} F_{l}^{+}\right)\left(\Delta^{+} F_{l}^{+} / / \sum_{l} \Delta^{+} F_{l}^{+}\right)^{2}=\left\langle\Delta^{-} F^{+}, \Delta^{+} F^{+}\right\rangle /\left\langle\Delta^{+} F^{+}, \Delta^{+} F^{+}\right\rangle$

(c) $r^{+}=\sum_{l}\left|\Delta^{-} F_{l}^{+}\right| \Delta^{-} F_{l}^{+}\left|\sum_{l}\right| \Delta^{+} F_{l}^{+}\left|\Delta^{+} F_{l}^{+}=\left\langle\left|\Delta^{-} F^{+}\right| \cdot \Delta^{-} F^{+}\right\rangle\right|\left\langle\Delta^{+} F^{+} \mid \cdot \Delta^{+} F^{+}\right\rangle$

(d) let

$$
r_{1}^{+}=r^{+} \text {of Eq. (3.28C) }
$$

and

$$
r^{+}= \begin{cases}0, & r_{1}^{+}<0 \\ \left(r_{1}^{+}\right)^{1 / 2}, & r_{1}^{+} \geq 0\end{cases}
$$

where <,> denotes inner product among all $\mathrm{m}$ components. Clearly, the first choice is simplest.

Now, since $\varphi^{ \pm}$are scalar functions, what is used for the scalar equation carries over automatically to systems. This completes the construction of TVD scheme Eq. (3.14) for systems of conservation laws.

Remark 1. - As in the scalar case (Section 2), we denote in $\mathrm{Eq} .(3.14)$

$$
\varphi_{j}^{ \pm}=\varphi\left(r_{j}^{ \pm}\right)
$$

Remark 2. - In the course of developing the present scheme, the assumption of $A$ being constant is necessary. For the nonlinear case, a formal generalization is made. Thus, the same integration scheme of Eq. (3.14) sti1l applies since it is the flux differences that are used and no transformation is needed for evaluating $r$ and $\varphi$. Hence, the present scheme is not limited to flux vector splittings (for example, Steger and Warming 12 and van Leer 73 ), the Roe-type flux difference splitting ${ }^{3}$ is equally applicable.

Remark 3. - We avoid matrix-vector multiplications as needed in Harten's scheme or a Riemanntype solver (See e.g., Osher and Chakravarthy ${ }^{4}$, Roe $^{3}$ ), but require the calculation of "+" and "-" fluxes. The construction of " + " and "- "flux differences is as easy as that of $\Delta^{ \pm} F$, the differences in whole flux. Also, the calculation required of $r^{ \pm}$and $\varphi^{ \pm}$is minimal once $\Delta^{ \pm} F^{ \pm}$ are formed; the amount of operations in Eq. (3.14) should be nearly twice as much as the conventional non-1VD, l.ax-wendroff scheme (without adding additional dissipation). Also the increase in storage varies with schemes, and is minimal for explicit schemes.

Remark 4. - We can write the numerical fluxes $\tilde{F}_{j \pm 1 / 2}$ for the system in the same way as the scalar case (Section 2). If fact, one merely replaces in Eqs. (2.32) to (2.35) the scalar flux with flux-vectors and a with the Jacobian matrix A.

Generalization to Euler Equations = Multidimensions and Steady Equations

In this section we apply what has been described in Section 3 toward the solutions of Euler equations in one- and two-space dimensions. To describe the extension, we consider the one-dimensional equation

$$
U_{t}+F_{X}(U)=0
$$

where

$$
\begin{gathered}
U=\left[\begin{array}{l}
\rho \\
\rho \mathrm{u} \\
\rho E
\end{array}\right], \quad \mathrm{F}=\left[\begin{array}{c}
\rho \mathrm{u} \\
\rho \mathrm{U}^{2}+p \\
(\rho E+p) u
\end{array}\right] \\
\mathrm{p}=(Y-1) \rho\left(E-0.5 u^{2}\right)
\end{gathered}
$$

To accomplish the differencing of $\mathrm{F}^{+}$and $F^{-}$in Eq. (3.14), we choose to use flux-vector splittings given by Steger-Warming 12 and van Leer 13 . They are written, for completeness, as follows: 


\section{(a) Steger-Warming}

Let the eigenvalues of $A$ be

$$
\left(a_{1}, a_{2}, a_{3}\right)=(u, u+c, u-c), c^{2}=\gamma p / \rho
$$

and

$$
a_{\ell}^{ \pm}=\left(a_{\ell} \pm\left|a_{\ell}\right|\right) / 2,1 \leq \ell \leq 3
$$

Since the flux vector in Eq. (4.1) is a homogeneous function of degree one, we have

$$
F=A U, \quad A=\partial F / \partial U
$$

Hence, the flux is split as

$$
F^{ \pm}=A^{ \pm} U=\frac{e}{2 r}\left[\begin{array}{l}
2(r-1) a_{1}^{ \pm}+a_{2}^{ \pm}+a_{3}^{ \pm} \\
2(r-1) a_{1}^{ \pm} a_{1}+a_{2}^{ \pm} a_{2}+a_{3}^{ \pm} a_{3} \\
(Y-1) a_{1}^{ \pm} a_{1}^{2}+0.5\left(a_{2}^{ \pm} a_{2}^{2}+a_{3}^{ \pm} a_{3}^{2}\right)+w
\end{array}\right]
$$

where $w=(3-\gamma)\left(a_{2}^{ \pm}+a_{3}^{ \pm}\right) c / 2(y-1)$.

\section{(b) van Leer}

To circumvent the discontinuity of first derivatives of $F \pm$ at transition states, $u=0$, or $u= \pm c$, van Leer constructs a flux-vector splitting consisting of a polynomial of degree two, thereby ensuring continuous first-order derivatives.

For supersonic flow, $|u|>c$

$$
\begin{array}{ll}
F^{+}=F, & u>0 \\
F^{-}=F, & u<0
\end{array}
$$

For subsonic flow, $|u|<c$, let $F_{1}^{ \pm}= \pm \rho(u \pm c)^{2} / 4 c$,

$$
F^{ \pm}=\left[\begin{array}{l}
F_{1}^{ \pm} \\
F_{1}^{ \pm}[(\gamma-1) u+2 c] / \gamma \\
F_{1}^{ \pm}[(\gamma-1) u \pm 2 c]^{2} / 2\left(\gamma^{2}-1\right)
\end{array}\right]
$$

Both splittings were used in the numerical experiments to determine their applicability to the oresent TVD scheme. In the following, we discuss the specific problems studied.

One-Dimensional Shock Tube Problem

10 test the performance of the second order accurate scheme Eq. (3.14), first we consider the Sod problem with the initial condition,

$$
(p, p, u)= \begin{cases}(10.0,8.0,0.0), & x<0 \\ (1.0,1.0,0.0), & x>0\end{cases}
$$

Figures 2 show the comparisons of calculated and exact solutions of pressure, density, internal energy, and velocity; the initial condition is also included. The Steger-Warming splitting was used; the CFL number was 0.95 and the doma in divided equaliy into 200 intervals. The limiter function was

$$
\varphi_{1}= \begin{cases}\min (1, r), & r>0 \\ 0, & r<0\end{cases}
$$

where $r$ was defined by Eq. (3.28c). It is evident that the contact discontinuity was smeared due to excessive diffusivity. Harten ${ }^{2}$ reported substantial improvement in the resolution of the contact discontinuity through the addition of artificial compression. Here we show the results of using different definitions given in Eqs. (3.28). (Note: the definition for $r^{-}$, although not given here, can be easily gotten from Eq. (3.20b) and by following steps Eqs. (3.21) to (3.28)). The definition of Eq. (3.28a), the results of which are shown in Figs. 3, clearly gave the best results while the results corresponding to $\mathrm{Eq}$. (3.28b) were the poorest, displaying as evident in Fig. 4 slight oscillations near the contact discontinuity. However, the shock wave and expansion waves were predicted very well for the nonlinear system in a 11 cases. Next Roe's "superbee"14 was used,

$\varphi_{2}=\left\{\begin{array}{cc}\max \{\min (2 r, 1), \min (r, 2)\}, & r>0 \\ 0, & r<0\end{array}\right.$

It is seen in Fig. 5 that the contact discontinuity was further improved. But slight oscillations occurred in the expansion region, possibly due to the fact of using $\Phi \leq 2$ which violates the TVD conditions in the present formulation. We also devised a mechanism by which a dramatic improvement was observed at the contact discontinuity; more analysis is underway and will be reported e] sewhere.

Next we tested a case involving a much stronger shock wave with the initial data:

$$
(p, \rho, u)= \begin{cases}(500,400,0.0), & x<0 \\ (1.0,1.0,0.0), & x>0\end{cases}
$$

Figures 6 show the results calculated using Eq. ( $3.28 \mathrm{a})$; the shock wave as well as the expansion waves were accurately predicted and monotonicity was satisfied. However, the extent of smearing at the contact discontinuity was not acceptable. Hence, calculations with finer meshes of 500 equal spacings were made, as seen in Figs. 7 much better results were obtained.

\section{Quasi_ One-Dimensional_ Nozzle Problem}

The flows are described by

$$
U_{t}+F_{x}(U)=H(U), \quad H(U)=\left[\begin{array}{l}
0 \\
p \\
0
\end{array}\right]
$$

where $S$ is the cross-sectional area of the nozzle. Two nozzle shapes were considered: 
$s_{1}(x)=1.398+0.347 \tanh (0.8 x-4), 0 \leq x \leq 10$

and

$S_{2}(x)=\left\{\begin{array}{l}1.75-0.75 \cos (0.2 x-1) \pi, 0 \leq x \leq 5 \\ 1.25-0.25 \cos (0.2 x-1) \pi, 5 \leq x \leq 10\end{array}\right.$

Uniform grids of 100 intervals were used in both nozzles. In a divergent channel where the inflow Mach number was 1.26 and the exit pressure ratio was 0.746 , the van Leer splitting Eq. (4.5) edged Steger-Warming's in resolving the shock wave, Fig. 8. This is related to the continuous transition of fluxes splitting through the sonic state inside the "numerical" shock wave. It is more evident in the convergent-divergent channel where the inflow Mach number and the exit pressure ratio are 0.2395 and 0.84 respectively. Figure 9 displays clearly the "kink" at the sonic throat in Sterger-Warming's splitting. The van Leer splitting performed admirably well at the throat although the exact solution has a discontinuity in first derivative resulting from a jump in $S_{2}$ ".

We also applied the same idea for constructing the above-described TVD scheme, to the MacCormack explicit method. The purpose was to sec whether and how well the present idea worked in a scheme where each of the predictor-corrector steps utilizes one-sided (but not upwind) differences. The calculated results, although not shown, were essentially identical in both nozzle flows.

\section{Two-Dimensfonal Problem}

A completeiy different approach toward the solution was taken: (1) Steady-state equations were used, i.e.

$$
F_{x}(U)+G_{y}(U)=0
$$

Here the type of the equations may be no longer hyperbolic and hence the notion of TVD is unclear; (2) We used Newton's method to linearize the system Eq. (4.12) and obtained an implicit integration procedure, as opposed to the Lax-Wendroff type; And finaliy, (3) The basic underlying scheme used second-order upwind differencing and was converted to a form similar to Eq. (3.12). For example, the derivative of $F$ is approximated by

$$
\begin{aligned}
F_{x}=\left\{\Delta^{-} F_{j}^{+}+\Delta^{+} F_{i}^{-}+\frac{1}{2}\right. & {\left[\Delta ^ { + } \left(\varphi_{j-1}^{+} \Delta^{-} F_{j-1}^{+}\right.\right.} \\
& \left.\left.\left.-\overline{\varphi_{j}^{-}} \Delta^{-} F_{j+1}^{-}\right)\right]\right\} / \Delta x
\end{aligned}
$$

Without mathematical guidance, we simply carry over the preceding formulation. However, it is assumed that since the time-asymplote steady solution to the unsteady equations can preserve monotonicity, the solution to the steady equations must also have the identical property (uniqueness of solutions is assumed).

In one-dimensional problems, the shock wave is aligned with a grid line and can be represented with a sharp profile by TVo schemes. In this section we wish to test our scheme on two-dimensional problems, in particular where the shock waves cut across grid lines. We consider a regular reflection of an oblique shock wave from a solid surface. Two inflow Mach numbers were tested to see the effect of shock strength on the performance. The inflow conditions were fully specified with freestream values and the conditions at the top boundary were set to satisfy the shock-jump relations with a specified shock angle. The variables at the outflow boundary were extrapolated linearly. At the solid wall, we let $v$ and the gradient of the other variables vanish. (See also ref. 11).

The first case had an incoming Mach number of 2.9 and a shock angle of 29.0 degrees; the computational domain contained $60 \times 20$ meshes equally divided in a domain $0.0<X<4.0$, and $0.0<Y<1.0$. Figures 10 show the comparisons of pressure from different calculations with exact solutions at $Y=0$ and 0.5 , together with pressure contours. The second-order upwind scheme with no added artificial-damping displayed over-expansion and over-compression just upstream and downstream of the shock, thereby causing slight irregularities in contours near the shock wave. All calculations with the present procedure did predict monotonic transition through shock waves, but in most cases the shock waves were smeared to a much larger extent than in the one-dimensional problems. The definition of Eq. (3.28a) gave slightiy better representation of shock waves than $\mathrm{Eq} .(3.28 \mathrm{C})$. The best came with using van Leer's splitting and Eq. (3.28a), showing much improved shock-wave resolutions, perhaps as good as from the onedimensional cases. This might look surprising since in the supersonic flows both splittings should give the identical results. However, this is true only for the $x$-flux $F$, but the $y$-flux $G$ is split differently as the transverse velocity component is subsonic.

Next let us consider a strong shock wave resulting in an overall pressure rise of a factor of 153; the incoming Mach number was 10.0 and the shock angle remained the same as above. We divided the domain $0.0<X<5.5$, and $0.0 \leq Y<1.0$ into $82 \times 20$ meshes with same $\Delta x$ as above. The van Leer splitting was used along with definition Eq. (3.28a). The pressure distributions and contours are given in Figs 11. The pressure at $Y=0.5$ was remarkably we 11 -behaved. However, the monotonicity seemed lost in the surface pressure, possibly due to extrapolation from the interior solutions. Roe's "superbee" displayed slightly sharper representation of shock waves than the case $\Phi \leq 1.0$, but also developed a stronger overshoot in the surface pressure just behind the shock wave.

\section{Concluding Remarks}

In the present paper we showed a simple and general procedure for constructing a TVD differencing scheme in which second-order accuracy was maintained, except in regions having extrema of fluxes. We showed that any non TVO differencings could be converted by writing it as a first-order upwind scheme plus remaining higher-order fluxes differences upon which limiting functions were applied so as to satisfy Harten's TVD conditions 
in the nonlinear scalar equation and constantcoefficient hyperbolic system of equations. A mathematical proof was given and definitions of arguments for the limiter functions derived for the system. Generalizations to the nonlinear Euler equations were carried out. Numerical tests were conducted to see the performance of the proposed scheme; questions regarding limiters were investigated. We found the definition of the argument appearing in a limiter was quite critical in getting good resolution for discontinuities, but otherwise all definitions seemed to yield monotone behavior. Further improvement in representing a contact discontinuity was also found possible. It has become clear to us that more analyses can be done in the area of limiters to achieve substantial improvements. We shall report this in a forthcoming paper ${ }^{15}$. It was also demonstrated that for steady-state calculations the van Leer splitting was in all cases more accurate than the Steger-Warming splitting.

\section{Acknowlegments}

The author would like to thank Or. H.C. Yee of NASA Ames Research Center for providing useful information when he had interests in this subject initially. Professor B. van Leer of the University of Michigan kindly sent his publications which were beneficial to this work.

\section{References}

1. van Leer, B, "Towards the Ultimate Conservative Difference Scheme II. Monotonicity and Conservation Combined in a Second-0rder Scheme," Journal of Computational Physics vol. 14, 1974, pp. 361-370.

2. Harten, A., "High Resolution Schemes for Hyperbolic Conservation Laws, "Journal of Computational Physics, Vol. 49, 1983, pp. 357-393.

3. Roe, P.L., "Approximate Riemann Solvers, Parameter Vectors, and Difference Schemes," Journal of Computation Physics, Vol. 43, 1981, pp. 357-372.

4. Osher, S., and Chakravarthy, S., "High Resolution Schemes and The Entropy Condition." SIAM Journal of Numerical Analysis, Vol. 21, No. 5 , Oct. 1984, pp. 955-984.

5. Sweby, P.K., "High Resolution Schemes Using Flux Limiters for Hyperbolic Conservation Laws," SIAM Journal of Numerical Analys is. Vol. 21, No. 5, 0ct. 1984, pp. 995-1011.
6. Boris, J.P. and Book, D.L., "Flux Corrected Transport, I, SHASTA, A Fluid Transport Algorithm That Works," Journal of Computational Physics, Vol. 11, 1973, Pp. 38-69.

7. Harten, A. and Lax, P.D., "A Random Choice Finite Difference Scheme for Hyperbolic Conservation Laws," SIAM Journal of Numerical Analys 15, Vol. 18, No. 2, Apr. 1981, pp. 289-315.

8. Harten, A., Engquist, B., Osher, S., and Chakravarthy, S., "Uniformly High Order Accurate Essentially Non-0scillatory Schemes 3," NASA CR-178101, 1986

9. Harten, A., Lax, P.D., and van Leer, B., "On Upstream Differencing and Godunov-Type Schemes for Hyperbolic Conservation Laws," SIAM Review, Vo1. 25, No. 1, Jan. 1983, pp. 35-61.

10. van Leer, B., "On the Relation between the Upwind-Differencing Schemes of Godunov, Engquist-0sher and Roe," SIAM Journal on Scientific and Statistical Computing, Vol. 5, No. 1, Mar. 1984, pp. 1-20.

11. Yee, H.C., Warming, R.F., and Harten, A. "Implicit Total Variation Diminishing (TVO) Schemes for Steady-State Calculations," Journal of Computational Physics, Vol. $57, \frac{1985}{9}$, pD. $327-360$

12 Steger, J.L. and Warming, R.F., "Flux Vector Splitting of the Inviscid Gasdynamic Equations with Application to Finite Difference Methods," Journal of Computational Physics, Vol. 40, 1981, pp. 263-293.

13. van Leer, B., "Flux-Vector Splitting for the Euler Equations, "International Conference on Numerical Methods in Fluid Dynamics, 8th, (Lecture Notes in Physics, Vol. 170) E. Krause, ed., Spriner-Verlag, New York, pp. 507-512.

14. Roe, P.L.. "Some Contributions to the Modelling of Discontinuous Flows," Large Scale Computations in Fluid Mechanics, (Lectures in Applied Mathematics, Vol. 22, Part II) B.E. Engquist, S. Osher and R.C.J. Somerville, eds., American Mathematical Society, Providence, 1985, pp. 163-193.

15. Liou, M.-S., "On Relation between Flux-Vector and Flux-Difference Splittings," in preparation. 

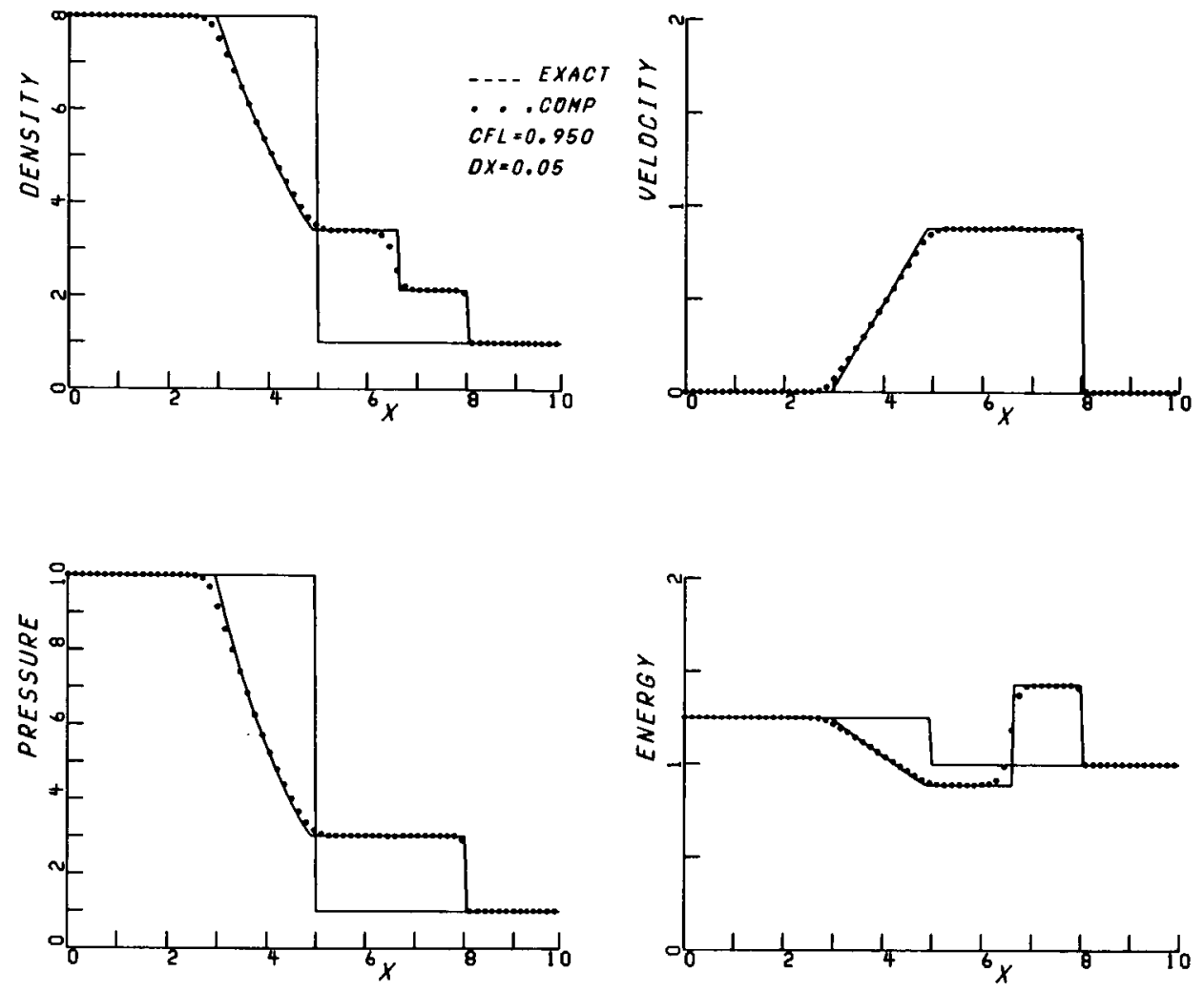

FIGURE 2. - ONE-DIMENSIONAL SHOCK TUBE PROBLEM. INITIAL CONDITION AS GIVEN IN EQ. (4.6). RESULTS FROM THE SOLUTION USING EQS. (3.28c) AND (4.7), $\Delta \mathrm{X}=0.05, \mathrm{CFL}=0.95$. 

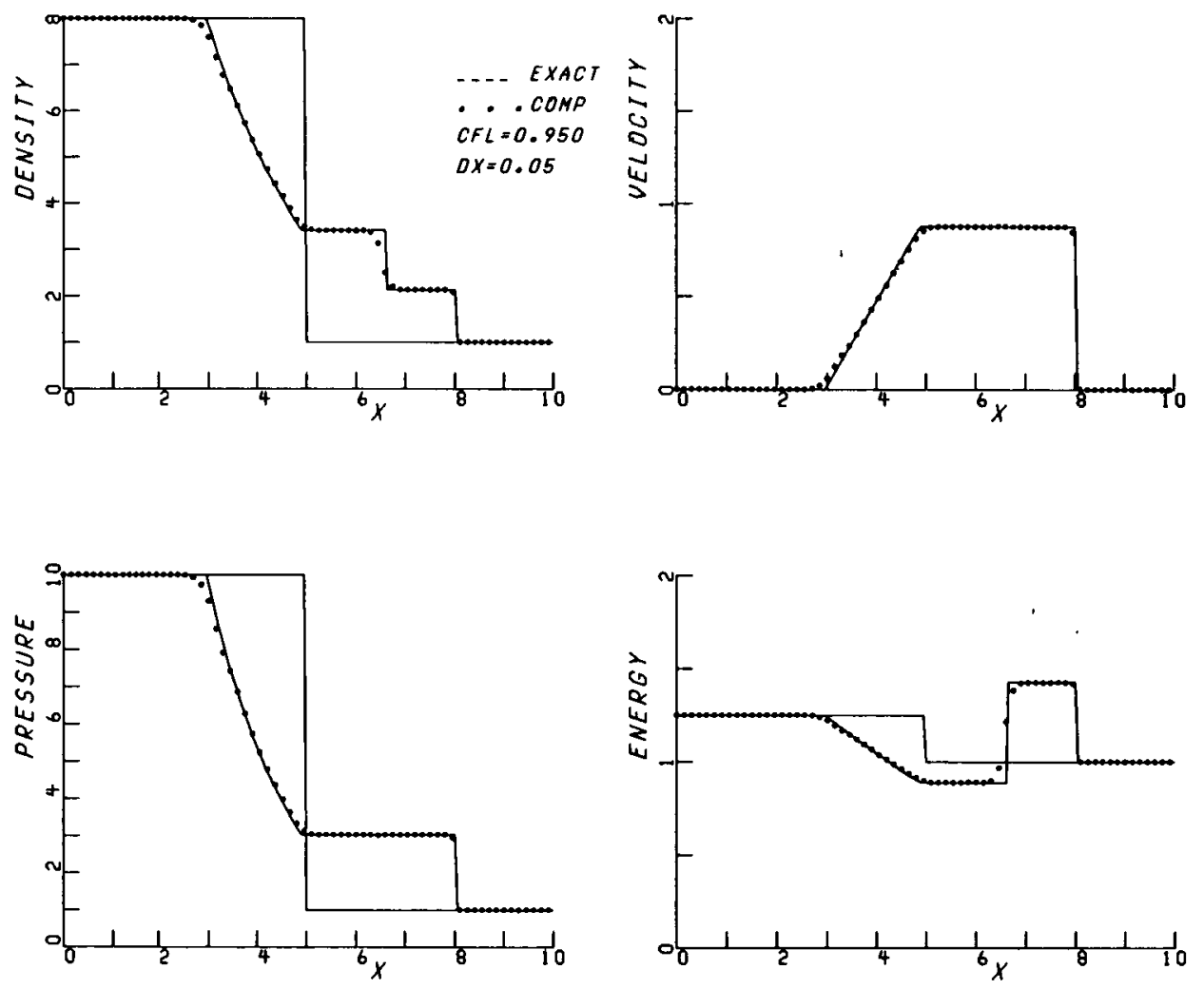

FIGURE 3. - ONE-DIMENSIONAL SHOCK TUBE PROBLEM. INITIAL CONDITION AS GIVEN IN EQ. (4.6). RESULTS FROM THE SOLUTION USING EQS. (3.28A) AND (4.7), $\Delta \mathrm{X}=0.05, \mathrm{CFL}=0.95$. 

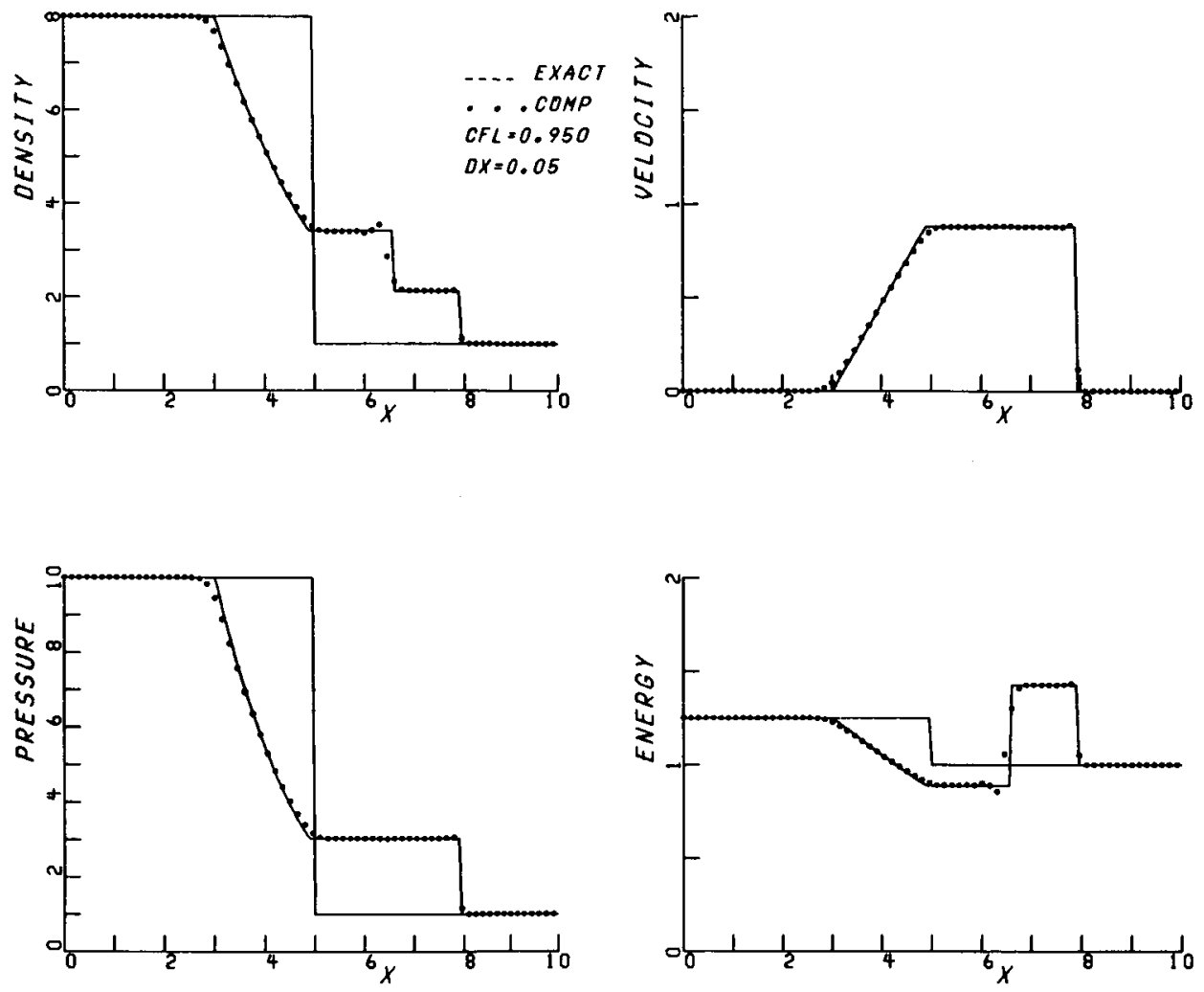

FIGURE 4. - ONE-DIMENSIONAL SHOCK TUBE PROBLEM, INITIAL CONDITION AS GIVEN IN EQ. (4.6). RESULTS FROM THE SOLUTION USING EQS. (3.28B) AND (4.7), $\Delta \mathrm{X}=0.05, \mathrm{CFL}=0.95$. 

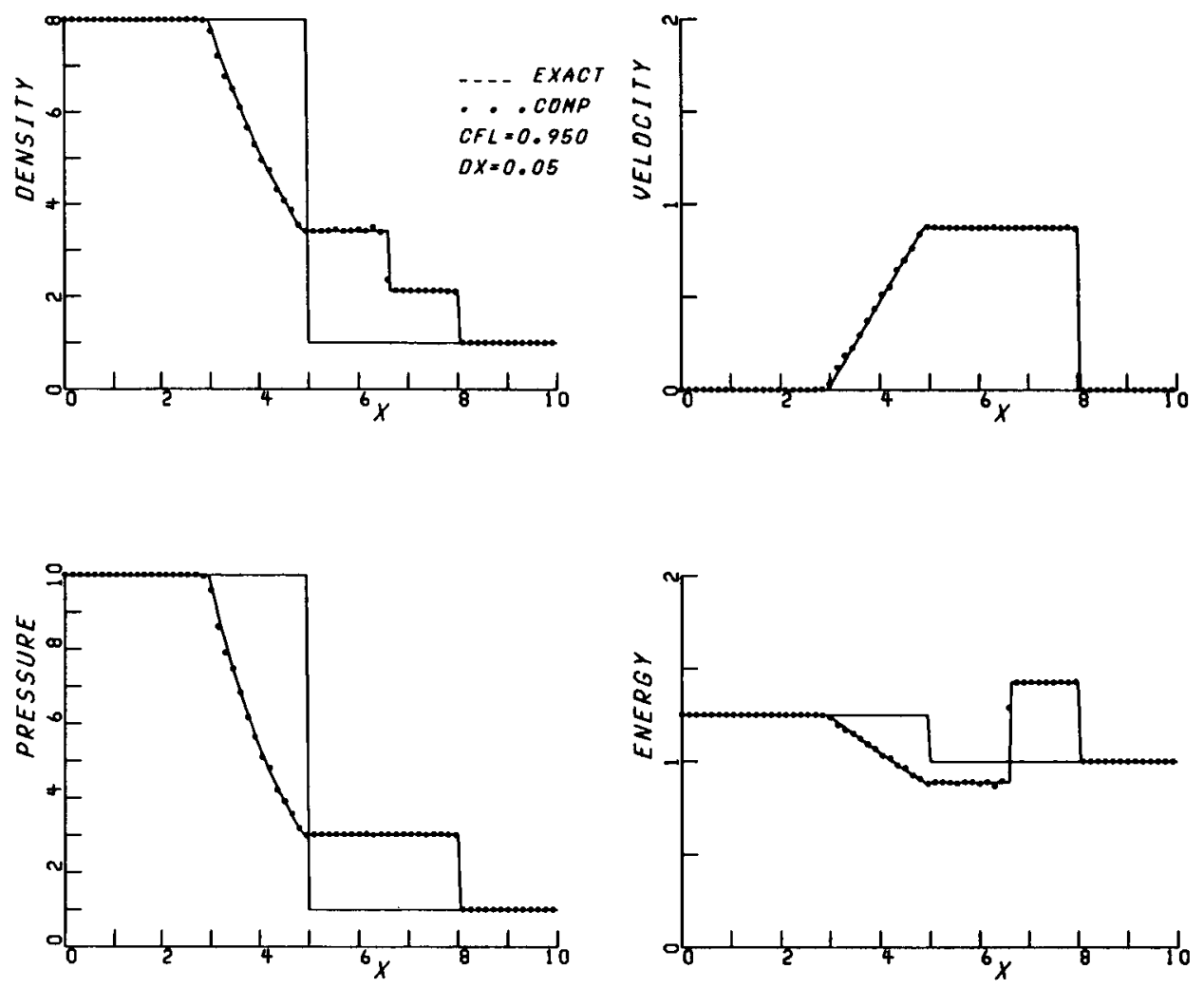

FIGURE 5. - ONE-DIMENSIONAL SHOCK TUBE PROBLEM, INITIAL CONDITION AS GIVEN IN EQ. (4.6). RESULTS FROM THE SOLUTION USING EQS. (328A) AND (4.8), $\Delta \mathrm{X}=0.05$. CFL $=0.95$. 

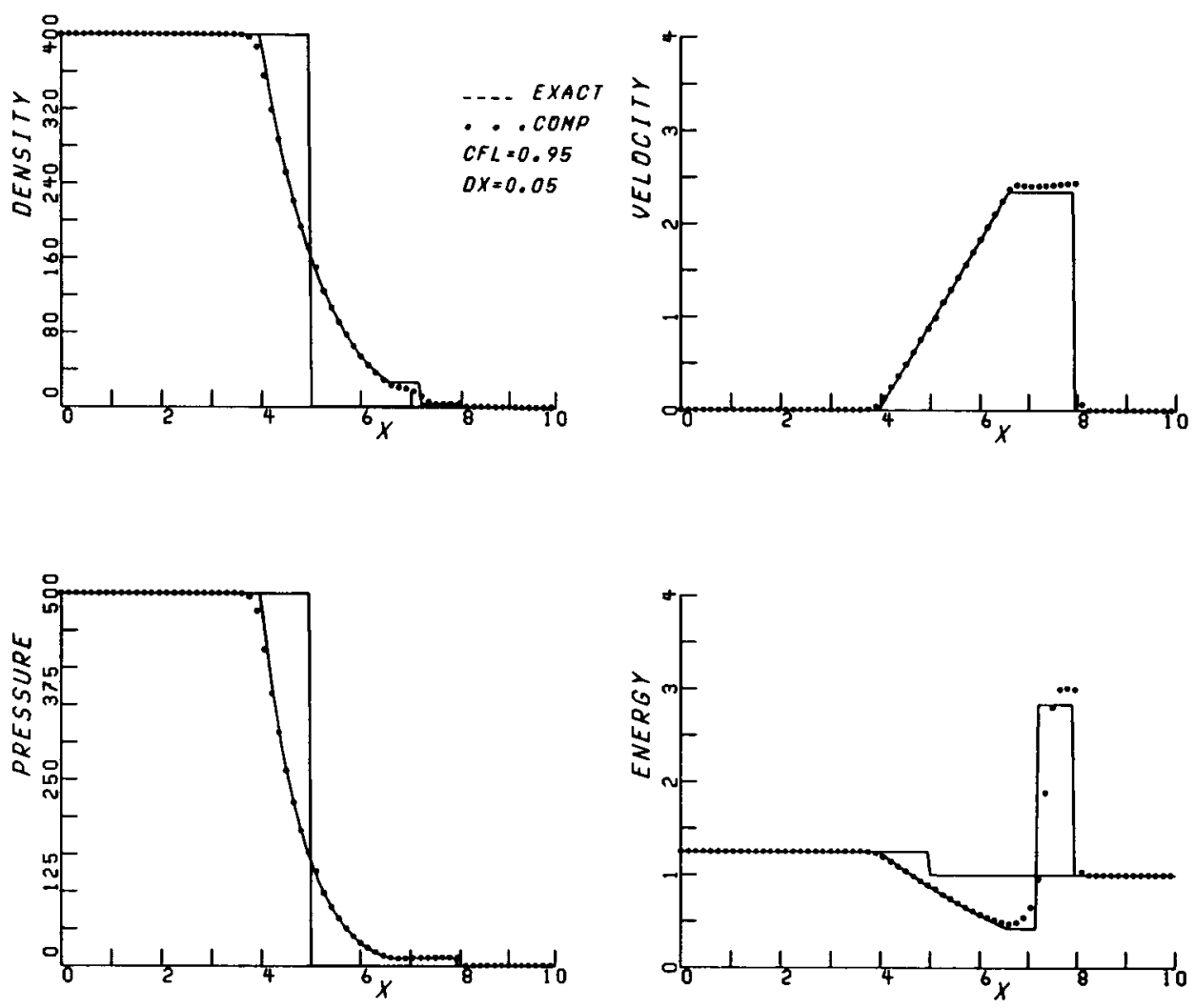

FIGURE 6. - ONE-DIMENSIONAL SHOCK TUBE PROBLEM, INITIAL CONDITION AS GIVEN IN EQ. (4.9). RESULTS FROM THE SOLUTION USING EQS. (3.28A) AND (4.7), $\Delta \mathrm{X}=0.05, \mathrm{CFL}=0.95$. 

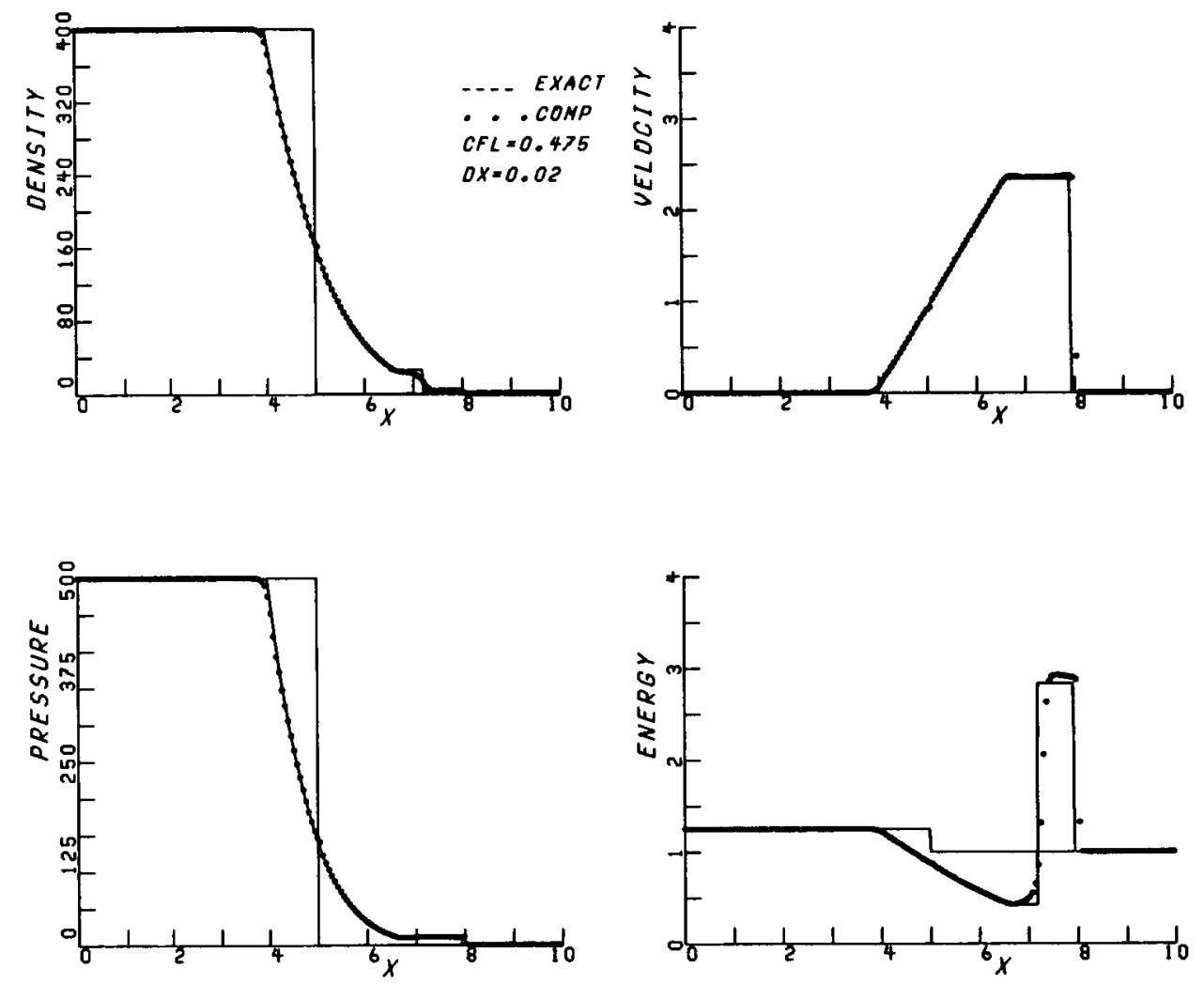

FIGURE 7. - ONE-DIMENSIONAL SHOCK TUBE PROBLEM, INITIAL CONDITION AS GIVEN IN EQ. (4.9). RESULTS FROM THE SOLUTION USING EQS. (328A) AND (4.7). $\Delta \mathrm{X}=0.02, \mathrm{CFL}=0.475$. 


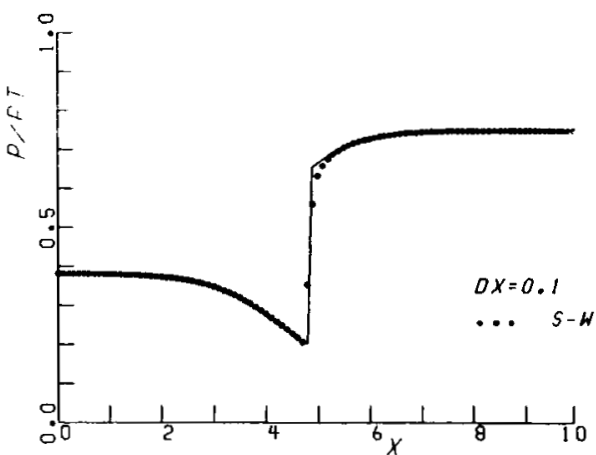

(A) STEGER-WARMING SPLITTING. EQS. (4.4).

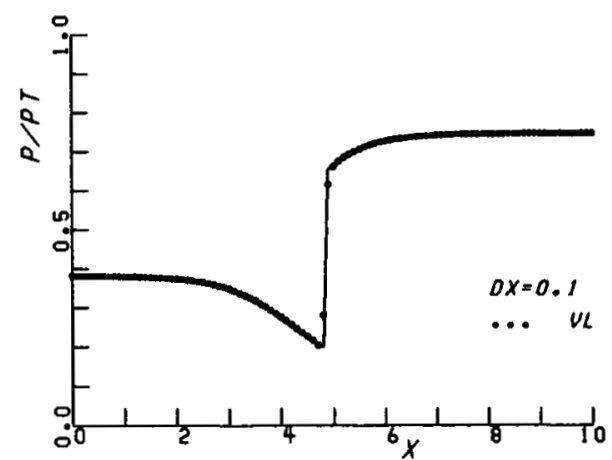

(B) VAN LEER SPLITTING, EQS. (4.5).

FIGURE 8, - STATIC PRESSURE DISTRIBUTION IN THE DIVERGENT NOZZLE, EQ. $(4.11 \mathrm{~A}), M_{\infty}=1.26$, RATIO OF STATIC TO TOTAL PRESSURE $=0.746$.

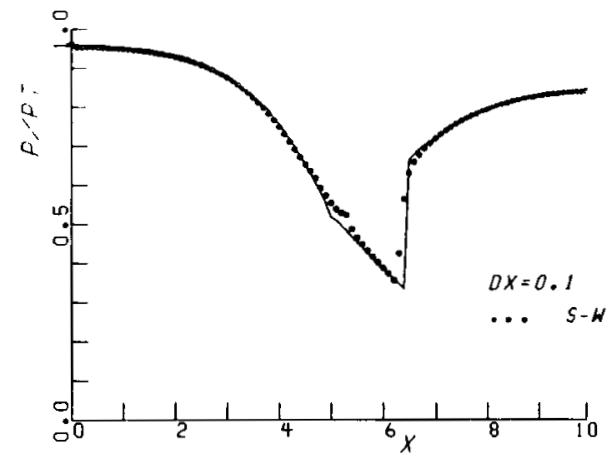

(A) STEGER-WARMING SPLITTING, EQS. (4.4).

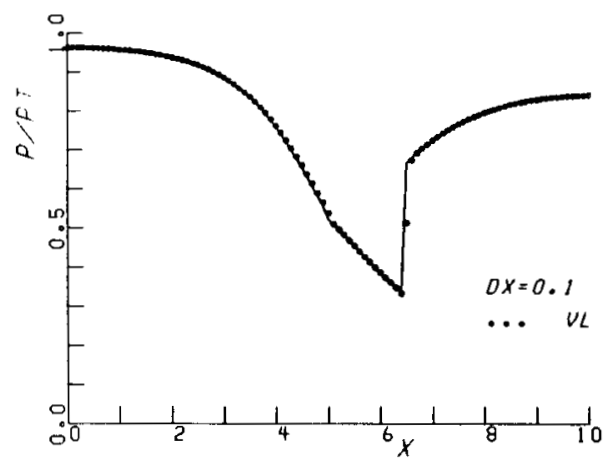

(B) VAN LEER SPLITTING, EQS. (4.5).

FIGURE 9. - STATIC PRESSURE DISTRIBUTION IN THE CONVERGENT-DIVERGENT NOZZLE, EQ. (4.11B), $M_{\infty}=0.2395$, RATIO OF STATIC TO TOTAL PRESSURE $=0.84$. 

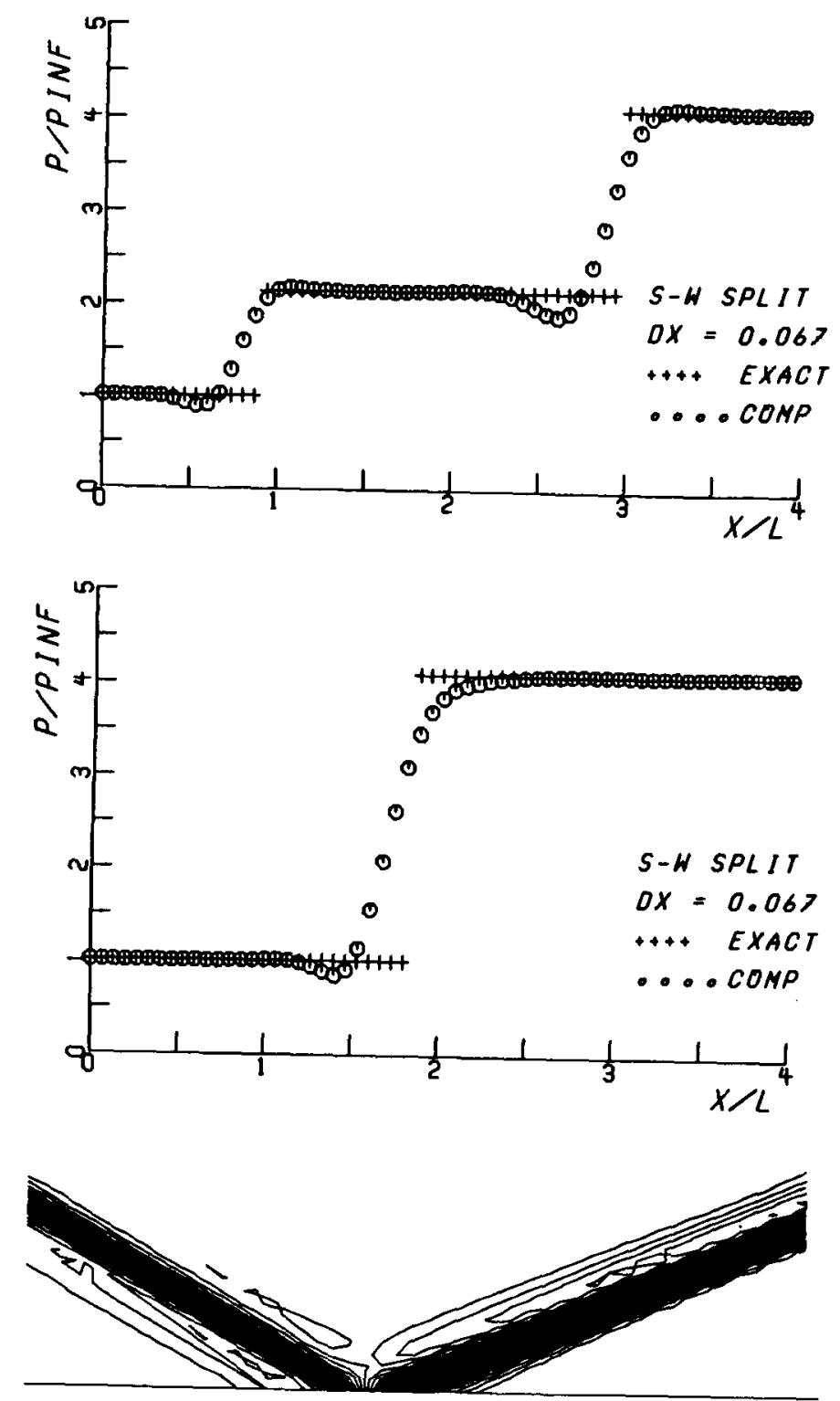

(A) SECOND-ORDER UPWIND SCHEME, SWS.

FIGURE 10. - SHOCK REFLECTION PROBLEM, STATIC PRESSURE DISTRIBUTION AT $y=0$ AND $y=0.5$ AND PRESSURE CONTOURS (MIN $=0.5$, MAX $=4.0$, INCREMENT $=0.007$ ). $M_{\infty}=2.9$. SHOCK ANGLE $=29$ DEGREES . 

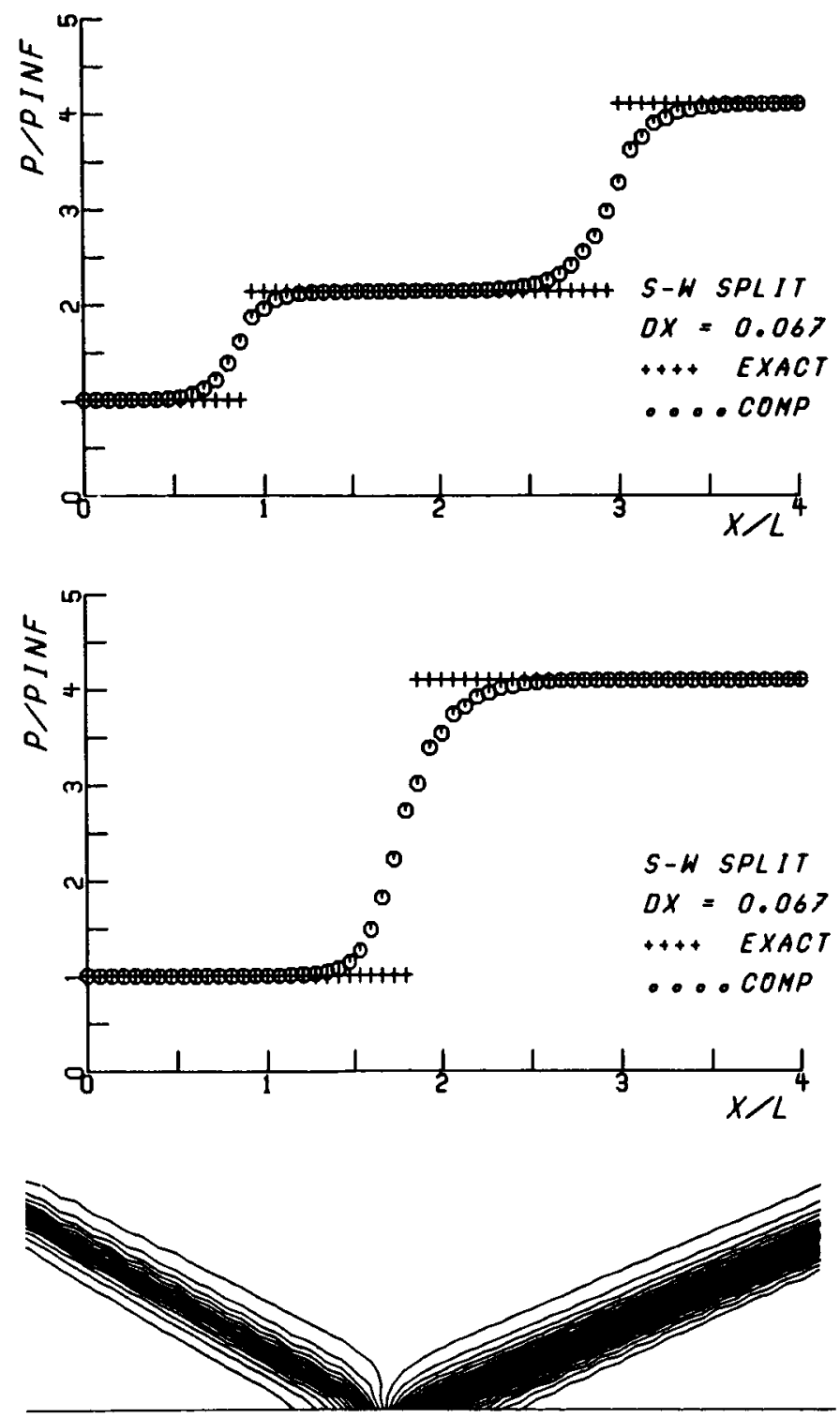

(B) EQS. (3.28C) AND (4.7), SWS. FIGURE 10. - CONTINUED. 

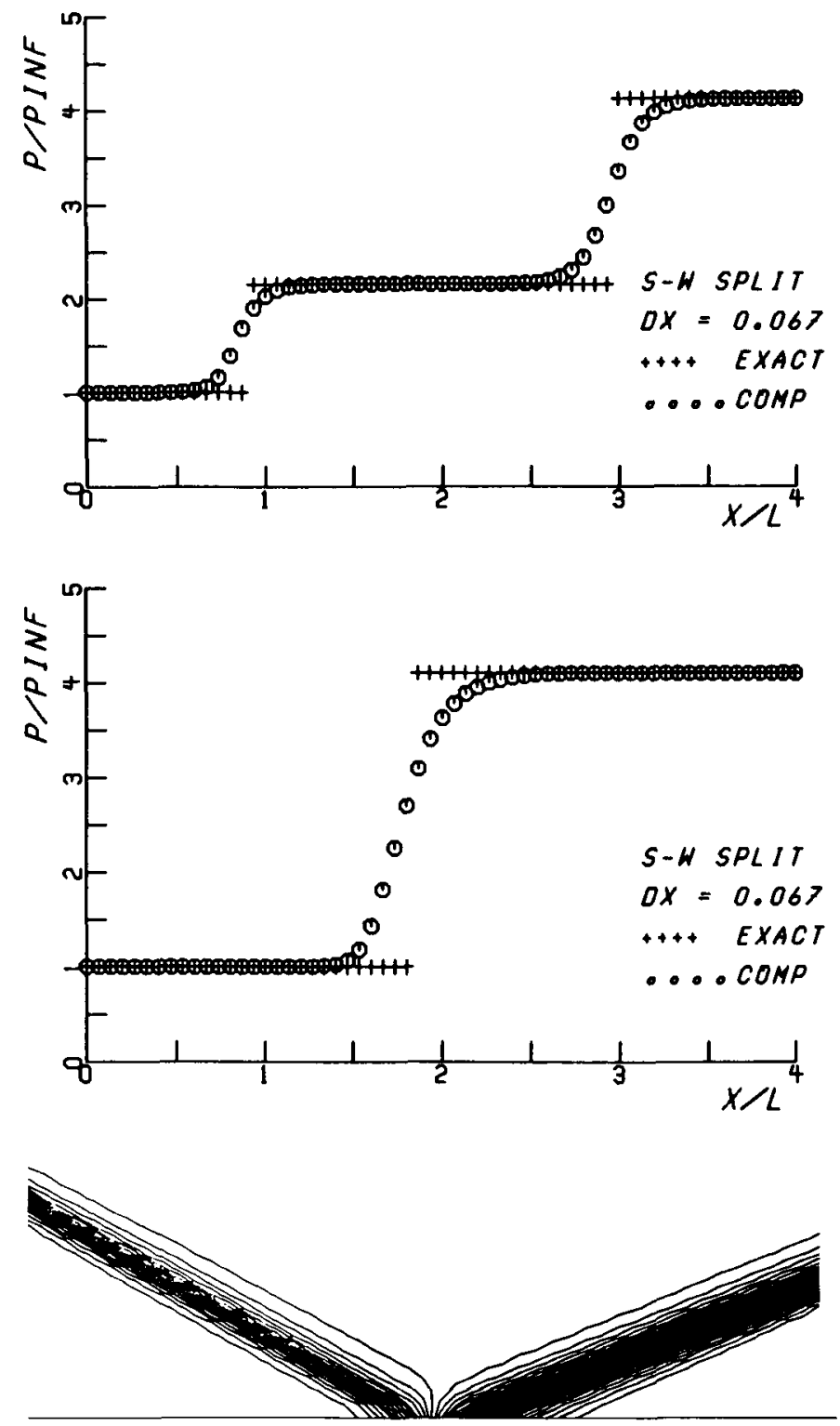

(C) EQS. (3.28A) AND (4.7), SWS.

FIGURE 10. - CONTINUED. 

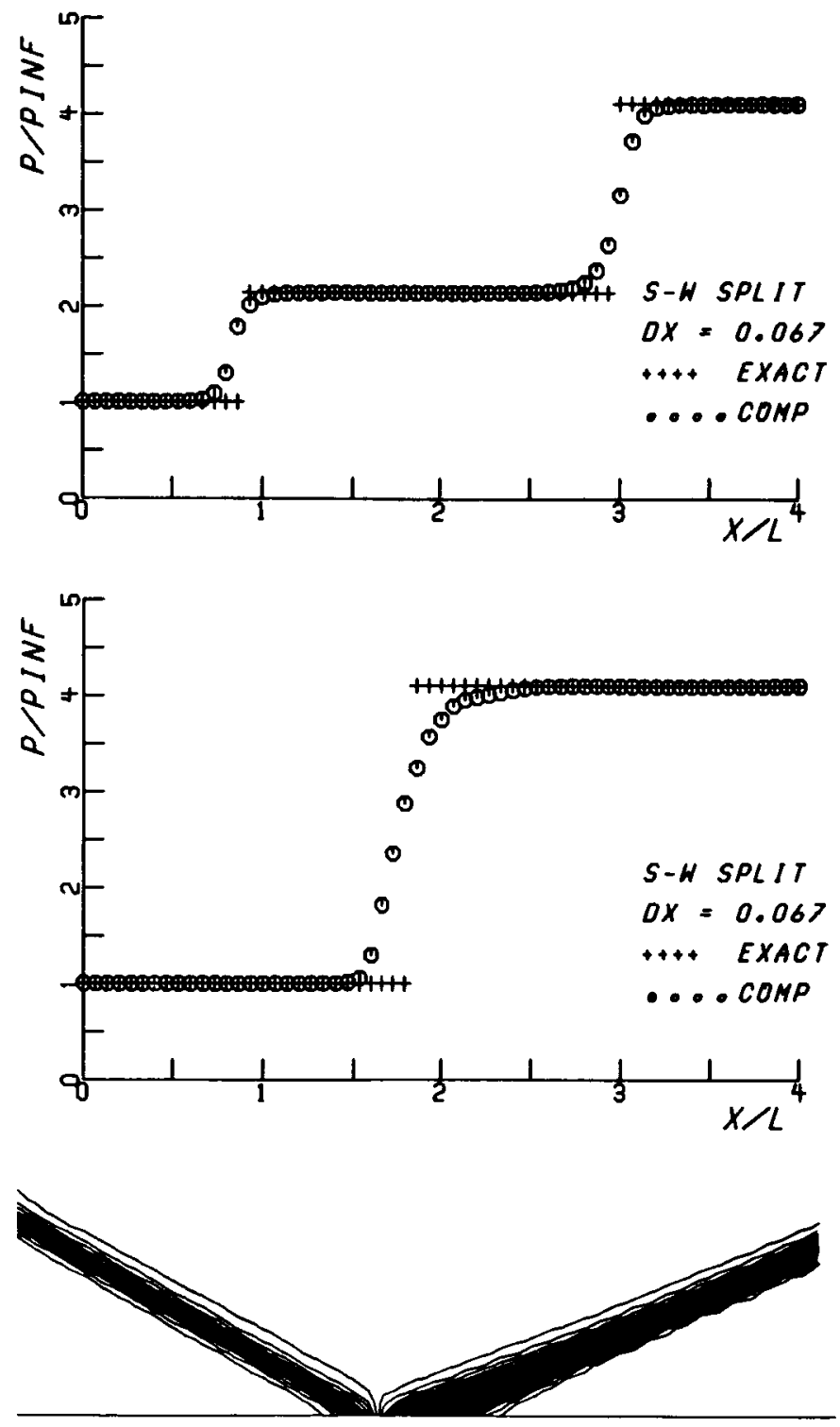

(D) EQS. (3.28A) AND (4.8), SWS.

FIGURE 10. - CONTINUED. 

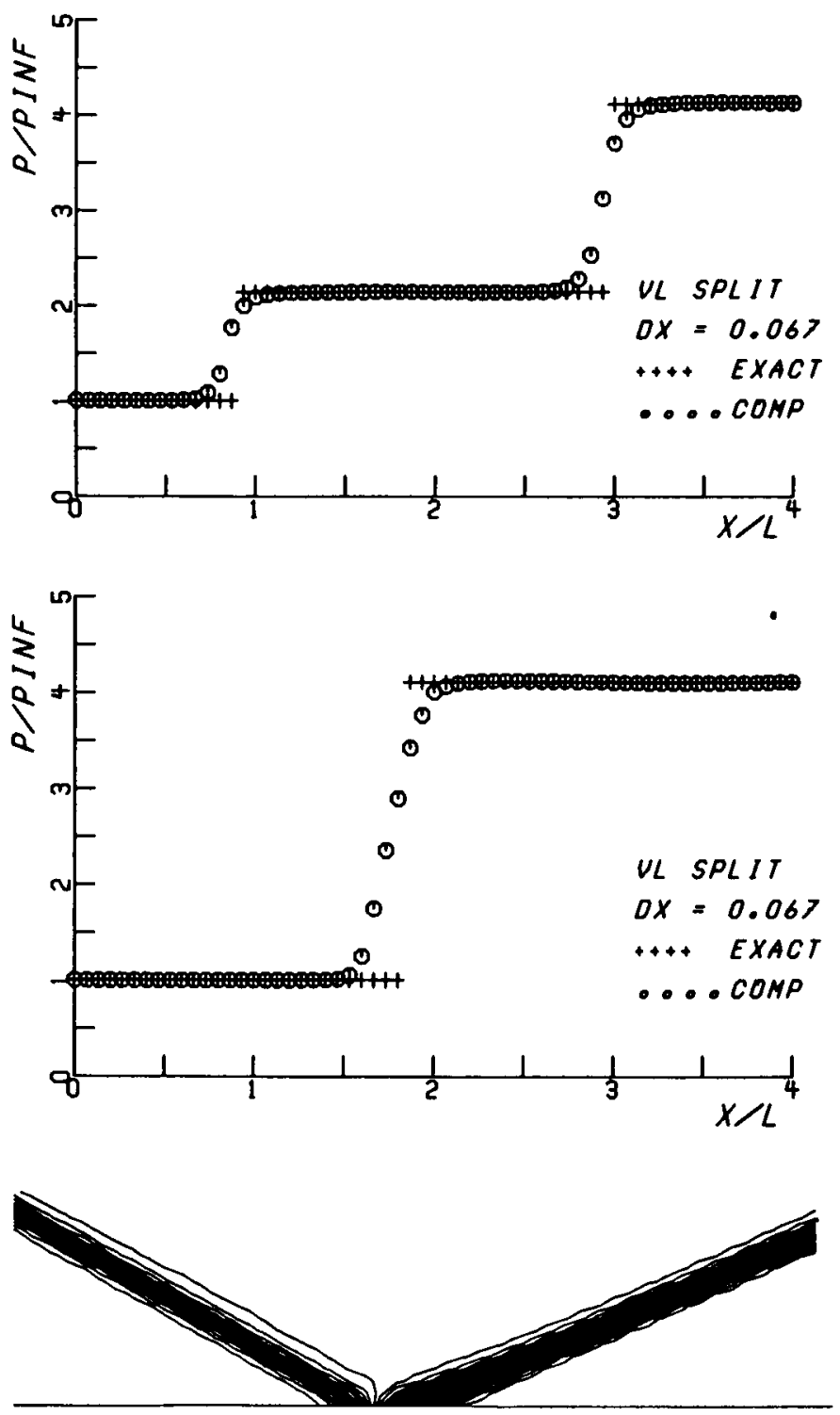

(E) EQS. (3.28A) AND (4.8), VLS.

FIGURE 10. - CONCLUDED. 

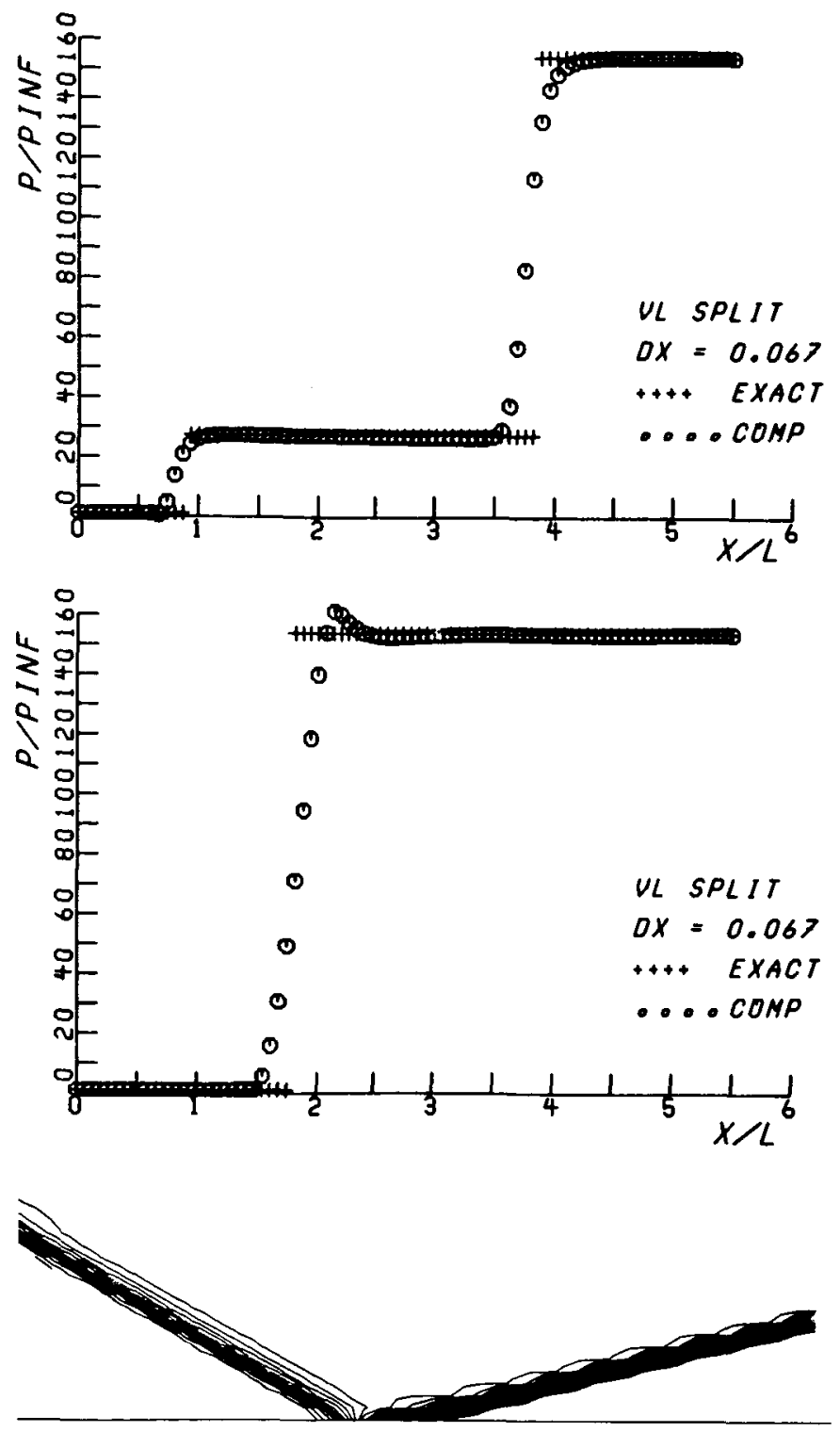

(A) EQS. (3.28A) AND (4.7), VLS.

FIGURE 11. - SHOCK REFLECTION PROBLEM, STATIC PRESSURE DISTRIBUTION AT $y=0$ AND $y=0.5$ AND PRESSURE CONTOURS (MIN $=0.5$, MAX $=150.0$. INCREMENT $=2.25)$, $M_{\infty}=10.0$ AND SHOCK ANGLE $=29$ DEGREES. 

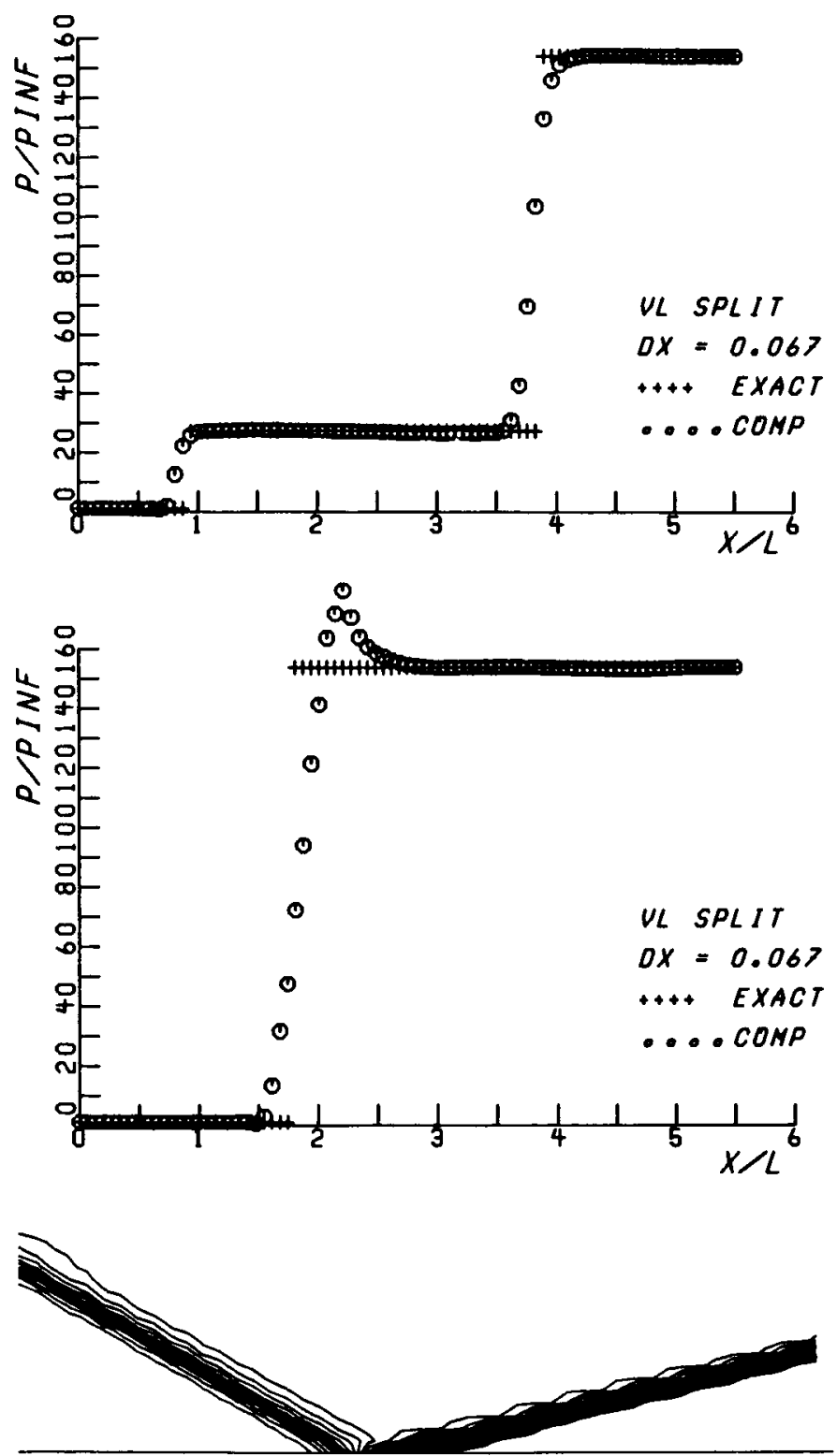

(B) EQS. (3.28A) AND (4.8), VLS.

FIGURE 11. - CONCLUDED. 


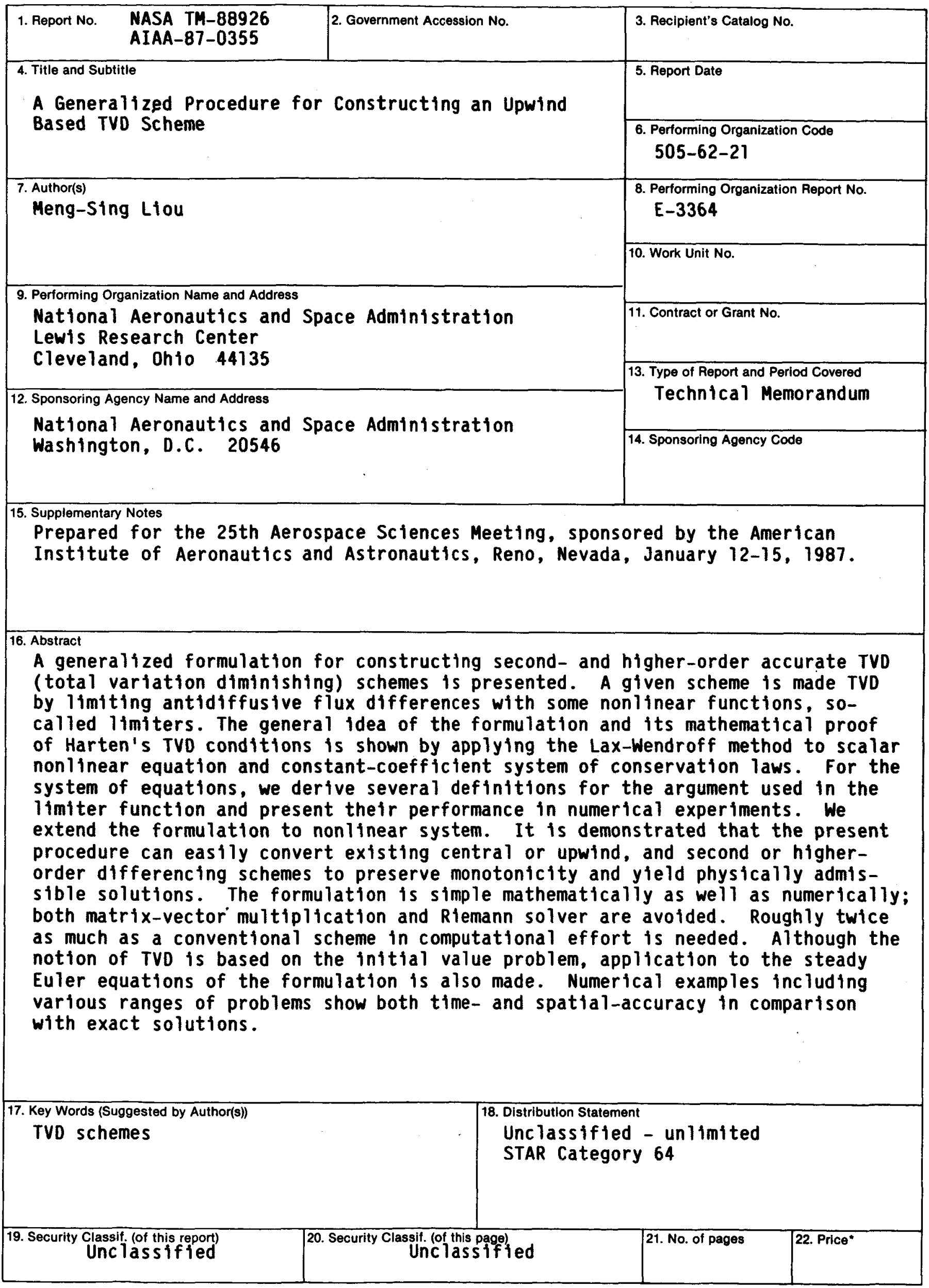

"For sale by the National Technical Information Service, Springfield, Virginia 22161 